ESAIM: COCV 21 (2015) 1053-1075

DOI: $10.1051 / \mathrm{cocv} / 2014058$
ESAIM: Control, Optimisation and Calculus of Variations

www.esaim-cocv.org

\title{
ON THE LOWER SEMICONTINUITY OF SUPREMAL FUNCTIONAL UNDER DIFFERENTIAL CONSTRAINTS
}

\author{
Nadia Ansini ${ }^{1,2}$ And Francesca Prinari ${ }^{3}$
}

\begin{abstract}
We study the weak* lower semicontinuity of functionals of the form

$$
F(V)=\operatorname{ess}_{x \in \Omega} f(x, V(x))
$$

where $\Omega \subset \mathbb{R}^{N}$ is a bounded open set, $V \in L^{\infty}\left(\Omega ; \mathbb{M}^{d \times N}\right) \cap \operatorname{Ker} \mathcal{A}$ and $\mathcal{A}$ is a constant-rank partial differential operator. The notion of $\mathcal{A}$-Young quasiconvexity, which is introduced here, provides a sufficient condition when $f(x, \cdot)$ is only lower semicontinuous. We also establish necessary conditions for weak* lower semicontinuity. Finally, we discuss the divergence and curl-free cases and, as an application, we characterise the strength set in the context of electrical resistivity.
\end{abstract}

Mathematics Subject Classification. 49J45, 35E99.

Received May 5, 2014.

Published online 24 June 2015.

\section{INTRODUCTION}

In this paper we study the lower semicontinuity of the $L^{\infty}$ functionals defined as

$$
F(V):=\operatorname{ess}_{x \in \Omega} \sup f(x, V(x)),
$$

where $\Omega \subset \mathbb{R}^{N}$ is a bounded open set, the function $f(x, \cdot)$ is lower semicontinuous for a.e. $x \in \Omega$ and $V \in$ $L^{\infty}\left(\Omega ; \mathbb{M}^{d \times N}\right) \cap \operatorname{Ker} \mathcal{A}$. That is, $V$ is constrained to satisfy a system of first-order linear partial differential equations:

$$
\mathcal{A} V:=\sum_{i=1}^{N} A^{(i)} \frac{\partial V}{\partial x_{i}}=0 .
$$

Here $A^{(i)}: \mathbb{M}^{d \times N} \rightarrow \mathbb{R}^{l}$ are linear transformations whenever $i=1, \ldots, N$ and the operator $\mathcal{A}$ satisfies the constant-rank condition (see [17]): there exists $r \in \mathbb{N}$ such that

\footnotetext{
Keywords and phrases. Supremal functionals, $\Gamma$-convergence, $L^{p}$-approximation, lower semicontinuity, $\mathcal{A}$-quasiconvexity.

1 Dip. di Matematica, Sapienza Università di Roma, P.le Aldo Moro 2, 00185 Rome, Italy. ansini@mat.uniroma1.it

2 Department of Mathematical Sciences, University of Bath, Claverton Down, Bath, BA2 7AY, UK

3 Dip. di Matematica e Informatica, Università di Ferrara, Via Machiavelli 35, 44121 Ferrara, Italy. prnfnc1@unife.it
} 
$\operatorname{rank} \mathbb{A} w=r \quad$ for all $w \in S^{N-1}$

where

$$
\mathbb{A} w=\sum_{i=1}^{N} A^{(i)} w_{i}, \quad w \in \mathbb{R}^{N} .
$$

This type of constraint arises naturally in the setting of continuum mechanics and electromagnetism: for example,

(a) in the case of solenoidal fields (divergence-free fields), which are relevant to treat extreme resistivity:

$$
\mathcal{A} V=0 \text { if and only if } \operatorname{Div} V=0,
$$

where $V: \Omega \rightarrow \mathbb{M}^{d \times N}$

(b) in the context of effective conductivity (curl-free fields):

$$
\mathcal{A} V=0 \text { if and only if curl } V=0 ;
$$

(c) in the micromagnetics literature when the constraints are given by Maxwell's equations;

(d) in the case of higher gradients.

(For further details, see ([15], Ex. 3.10)). The class of functionals represented in the "supremal" form (1.1) has been introduced to model physical situations where the relevant "quantities" are not negligible on sets of arbitrarily small measure (see e.g. $[1,5,12]$ ). In these cases it may be natural to formulate the problem as a minimum of a supremal functional as, for example, in [16] where the authors study dielectric breakdown for composites made of two homogeneous phases. In this case the corresponding variational principle is given by the minimum problem of a supremal functional of the form

$$
F(v):=\left\{\begin{array}{cc}
\operatorname{ess} \sup |\lambda(x) D v(x)| & \text { if } v \in W^{1, \infty}(\Omega), \\
+\infty & \text { otherwise }
\end{array}\right.
$$

where $\lambda(x)$ is a piecewise-constant function (constant values of which represent the two phases) and $D u$ satisfies the condition $\int_{Q} D u \mathrm{~d} x=\xi$, which corresponds to assigning the average electric field. In order to study the macroscopic behaviour of the two-phase composite material for the first failure dielectric breakdown model, the authors use an $L^{p}$-approximation approach and show that the supremal functional $\operatorname{ess}_{\sup } \operatorname{su}_{x \in \Omega}|\lambda(x) D v(x)|$ can be obtained as the $\Gamma$-limit of power-law functionals $F_{p}: L^{1}(\Omega) \rightarrow[0,+\infty]$ given by

$$
F_{p}(v):=\left\{\begin{array}{cl}
\left(\int_{\Omega}|\lambda(x) D v(x)|^{p} \mathrm{~d} x\right)^{1 / p} & \text { if } v \in W^{1, p}(\Omega), \\
+\infty & \text { otherwise }
\end{array}\right.
$$

as $p \rightarrow+\infty$ with respect to the $L^{1}$-topology.

The study of $L^{p}$-approximation, by way of $\Gamma$-convergence, has been addressed for more general functionals by Champion et al. in [13] (see also [19]) and in the framework of a constant-rank operator $\mathcal{A}$ by Bocea and Nesi in [8] and by the authors in [3].

Since the $\Gamma$-limit is always lower semicontinuous, the approach by $L^{p}$-approximation of a supremal functional can be very useful for studying the lower semicontinuity of supremal functionals.

In [3] we introduce the notion of an $\mathcal{A}-\infty$ quasiconvex function (see Def. 2.7) and we prove, among other results, that if $f: \Omega \times \mathbb{R}^{N} \rightarrow[0,+\infty)$ is a Carathéodory function, $\mathcal{A}-\infty$ quasiconvex in the second variable and satisfying a linear growth condition, then

$$
F(V):=\left\{\begin{array}{cl}
\underset{x \in \Omega}{\operatorname{ess} \sup } f(x, V(x)) & \text { if } V \in L^{\infty}\left(\Omega ; \mathbb{M}^{d \times N}\right) \cap \operatorname{Ker} \mathcal{A}, \\
+\infty & \text { otherwise }
\end{array}\right.
$$


is the $\Gamma\left(w^{*}-L^{\infty}\right)$-limit of the family of power-law functionals $F_{p}: L^{\infty}\left(\Omega ; \mathbb{M}^{d \times N}\right) \rightarrow[0,+\infty)$ given by

$$
F_{p}(V):=\left\{\begin{array}{cl}
\left(\int_{\Omega} f^{p}(x, V(x)) \mathrm{d} x\right)^{1 / p} & \text { if } V \in L^{\infty}\left(\Omega ; \mathbb{M}^{d \times N}\right) \cap \operatorname{Ker} \mathcal{A}, \\
+\infty & \text { otherwise }
\end{array}\right.
$$

as $p \rightarrow+\infty$ (see [3], Thm. 4.2).

Therefore in the continuous setting the notion of $\mathcal{A}-\infty$ quasiconvexity provides a sufficient condition for the $L^{\infty}$-weak* lower semicontinuity of the supremal functional under a differential constraint as in (1.1). However, $\mathcal{A}-\infty$ quasiconvexity is far from a necessary condition. In fact, in ([3], Example 3) we show that the class of curl$\infty$ quasiconvex functions is contained strictly in the class of strong Morrey quasiconvex functions (introduced by Barron, Jensen and Wang in [7] to prove the lower semicontinuity of a supremal functional defined on $\left.W^{1, \infty}\left(\Omega, \mathbb{M}^{d \times N}\right)\right)$. In addition, the continuity assumption on $f(x, \cdot)$, which is more natural in the context of integral functionals, is too restrictive for supremal functionals. In fact, if $f(x, \cdot)$ is lower semicontinuous and level convex (see (2.20)) then we still find that the functional (1.1) is $L^{\infty}$-weak* lower semicontinuous (see [1], Rem. 4.4) even if $f(x, \cdot)$ is not $\mathcal{A}-\infty$ quasiconvex (see [3], Example 5.9). On the other hand, the level convexity cannot to be considered a fulfilling condition for the vectorial case (see Example 6.6). For all these reasons the study of the lower semicontinuity of supremal functionals of the form (1.1) requires the introduction of a larger class of functions, containing both $\mathcal{A}-\infty$ quasiconvex and level convex functions.

To this end we define the class of $\mathcal{A}$-Young quasiconvex functions. We say that a function $f: \mathbb{M}^{d \times N} \rightarrow \mathbb{R}$ is $\mathcal{A}$-Young quasiconvex if it satisfies a generalised Jensen's inequality for Young measures; that is,

$$
f\left(\int_{\mathbb{M}^{d \times N}} \Sigma \mathrm{d} \mu_{x}(\Sigma)\right) \leq \underset{x \in Q}{\operatorname{ess} \sup } \mu_{x^{-}} \underset{\Sigma \in \mathbb{M}^{d \times N}}{\operatorname{ess} \sup } f(\Sigma)
$$

for a.e. $x \in Q$ and for every $\mathcal{A}$ - $\infty$-Young measure $\mu=\left(\mu_{x}\right)_{x \in Q}$ (see $\left.[4,9,15,22]\right)$.

This class of functions is not empty. In fact, thanks to ([7], Thm. 1.2), any lower semicontinuous and level convex function satisfies inequality (1.5). Moreover, in Proposition 3.4, we show that any continuous and $\mathcal{A}-\infty$ quasiconvex function is $\mathcal{A}$-Young quasiconvex. In Example 6.7 we construct a curl-Young quasiconvex function which is neither curl- $\infty$ quasiconvex nor level convex. Therefore the class of the $\mathcal{A}$-Young quasiconvex functions contains the class of level convex functions and the class of continuous $\mathcal{A}-\infty$ quasiconvex functions, but it is strictly larger. However, we show that, if $d=1, \mathcal{A}=\operatorname{curl}$ or $\mathcal{A}=\operatorname{div}$, then a lower semicontinuous and $\mathcal{A}$-Young quasiconvex function is level convex (see Prop. 6.4).

In Theorem 4.2 we prove that, if $f(x, \cdot)$ is lower semicontinuous and $\mathcal{A}$-Young quasiconvex for a.e. $x \in \Omega$, then the supremal functional $F$ given by (1.1) is weakly* lower semicontinuous on $L^{\infty}\left(\Omega ; \mathbb{M}^{d \times N}\right) \cap \operatorname{Ker} \mathcal{A}$. It is still an open question whether $\mathcal{A}$-Young quasiconvexity is also necessary. In Theorem 5.1 we provide some necessary conditions. More precisely, we show that if $F$ is $L^{\infty}$-weakly* lower semicontinuous and $f(\cdot, \Sigma)$ is continuous, then the supremand function satisfies

$$
f\left(x, t \Sigma_{1}+(1-t) \Sigma_{2}\right) \leq \max \left\{f\left(x, \Sigma_{1}\right), f\left(x, \Sigma_{2}\right)\right\}
$$

for every $x \in \Omega, t \in[0,1]$ and $\left(\Sigma^{1}-\Sigma^{2}\right) \in \Lambda$, where

$$
\Lambda:=\bigcup_{w \in S^{N-1}} \operatorname{Ker} \mathbb{A}(w)
$$

We also prove that $f(x, \cdot)$ is $\mathcal{A}$-weak quasiconvex (see Def. 2.8).

If $\mathcal{A}=$ curl, then $\mathcal{A}$-weak quasiconvexity coincides with weak Morrey quasiconvexity (see [3], Rem. 3.2(2)). We recall that, in the scalar case, weak Morrey quasiconvex functions are strong Morrey quasiconvex functions (see $[7,21])$. In a forthcoming paper [20] we will show that, in the vectorial case, the two classes do not coincide; this proves the conjecture raised by Barron et al.. 
In Section 6 we discuss Theorems 4.2 and 5.1 in the case of $\mathcal{A}=$ curl and $\mathcal{A}=$ Div. More precisely, when $\mathcal{A}=$ curl, Theorem 4.2 improves both ([7], Thm. 2.6) and ([13], Thm 3.4) since we do not require the continuity and coercivity of $f(\cdot, \Sigma)$. In Proposition 6.1 we show that curl-Young quasiconvex functions are strong Morrey quasiconvex functions. Conversely, in Proposition 6.3 we prove that polylevelconvex functions, which are introduced in ([7], Def. 3.5) as a subclass of strong Morrey quasiconvex functions, are curl-Young quasiconvex functions. Finally, since in the curl-free case we have

$$
\operatorname{Ker} \mathbb{A}(w)=\left\{\xi \otimes w \in \mathbb{M}^{d \times N}: \xi \in \mathbb{R}^{d}, w \in S^{N-1}\right\},
$$

it follows by Theorem 5.1 that, if $f(x, \cdot)$ is lower semicontinuous and curl-Young quasiconvex, then $f(x, \cdot)$ is rank-1 level convex; that is, $f$ satisfies (1.6) for every $\Sigma_{1}, \Sigma_{2} \in \mathbb{M}^{d \times N}$ with $\operatorname{rank}\left(\Sigma^{1}-\Sigma^{2}\right) \leq 1$. In the cases of $N=1$ or $d=1$ we find that the class of curl-Young quasiconvex functions coincides with the class of level convex ones (see Prop. 6.5).

In the case of $\mathcal{A}=\operatorname{Div}$, if $d=1$, then $\Lambda=\mathbb{R}^{N}$ and we find that the class of div-Young quasiconvex functions coincides with that of level convex ones. The vectorial case $(d \geq N>1)$ can be treated by generalising the scalar case; hence, if $f: \mathbb{M}^{d \times N} \rightarrow[0,+\infty)$ is lower semicontinuous and Div-Young quasiconvex, then $f$ is rank- $(N-1)$ level convex.

In Section 6.2 we characterise, by way of $\Gamma$-convergence, the effective strength set $K_{\text {eff }}$ in the context of electrical resistivity (that is, of how strongly a given material opposes the flow of electric current). This set is defined by

$$
K_{\text {eff }}:=\left\{\bar{\sigma}:=\int_{\Omega} \sigma(x) \mathrm{d} x: \sigma(x) \in K(x) \text { a.e. } x \in \Omega, \operatorname{div} \sigma=0\right\},
$$

with $K(x)=\left\{\xi \in \mathbb{R}^{N}: f(x, \xi) \leq 1\right\}$ and $f: \Omega \times \mathbb{R}^{N} \rightarrow \mathbb{R}$ is a Borel function that is lower semicontinuous and is level convex with respect to $\xi$ and satisfies a growth condition. In this model $\sigma: \Omega \mapsto \mathbb{R}^{N}$ is the current and the right differential constraint is the divergence. In ([8], Props. 6.1,6.2), Bocea and Nesi characterise the set $K_{\text {eff }}$ under the assumption that $f(x, \cdot)$ is convex. In this paper we improve their result by assuming that $f(x, \cdot)$ is lower semicontinuous and div-Young quasiconvex.

\section{Notation AND PRELIMINARIES}

Let $\Omega$ be a bounded open subset of $\mathbb{R}^{N}$. We denote by $\mathcal{O}(\Omega)$ the family of open subsets of $\Omega$. We write $\mathcal{L}^{N}(E)$ for the Lebesgue measure of $E \subset \mathbb{R}^{N}$. Let $\Sigma \in \mathbb{M}^{d \times N}$, where $\mathbb{M}^{d \times N}$ stands for the space of $d \times N$ real matrices, with a slight abuse of notation, we denote $|\Sigma|=\sum_{i=1}^{d}\left|\Sigma_{i}\right|$, where $\Sigma_{i}$ is the $i$ th row of $\Sigma$ and $\left|\Sigma_{i}\right|$ its Euclidean norm. We use $\xi_{i}$ also to denote the $i$ th component of a vector $\xi$. If $V: \Omega \rightarrow \mathbb{M}^{d \times N}$, we define $\operatorname{Div} V: \Omega \mapsto \mathbb{R}^{d}$ as $(\operatorname{Div} V)_{i}:=\operatorname{div} V_{i}$ whenever $i=1, \ldots, d$. Hereafter in this paper, we assume that $\mathcal{A}: L^{p}\left(\Omega ; \mathbb{M}^{d \times N}\right) \rightarrow W^{-1, p}\left(\Omega ; \mathbb{R}^{l}\right), 1<p \leq \infty$, is a constant-rank first-order linear partial operator given by

$$
\mathcal{A} V:=\sum_{i=1}^{N} A^{(i)} \frac{\partial V}{\partial x_{i}}
$$

where $A^{(i)}: \mathbb{M}^{d \times N} \rightarrow \mathbb{R}^{l}$ are linear transformations whenever $i=1, \ldots, N$. We denote the kernel of the operator $\mathcal{A}$ by

$$
\operatorname{Ker} \mathcal{A}:=\left\{V \in L^{p}\left(\Omega ; \mathbb{M}^{d \times N}\right): \mathcal{A} V=0\right\} .
$$

We recall that an $\mathcal{A}$ - $\infty$-Young measure is a measure generated by a sequence in $L^{\infty}\left(\Omega ; \mathbb{M}^{d \times N}\right) \cap \operatorname{Ker} \mathcal{A}$ weakly* converging in $L^{\infty}$ (see [15], Sect. 2). We denote the $N$-dimensional unit cube $(0,1)^{N}$ by $Q$. 


\section{1. $\mathcal{A}$-quasiconvexity}

In this section we recall some definitions and results we will use in the sequel.

Definition 2.1. Let $f: \mathbb{M}^{d \times N} \mapsto \mathbb{R}$ be a function. We say that $f$ is $\mathcal{A}$-quasiconvex if

$$
f(\Sigma) \leq \int_{Q} f(\Sigma+V(x)) \mathrm{d} x
$$

for every $V \in C_{\#}^{\infty}\left(\mathbb{R}^{N} ; \mathbb{M}^{d \times N}\right)$ such that $\mathcal{A} V=0$ and $\int_{Q} V \mathrm{~d} x=0$.

We denote by $C_{\#}^{\infty}\left(\mathbb{R}^{N} ; \mathbb{M}^{d \times N}\right)$ the set of $C^{\infty}\left(\mathbb{R}^{N} ; \mathbb{M}^{d \times N}\right)$ functions which are $Q$-periodic.

Definition 2.2. Given a Borel function $f: \mathbb{M}^{d \times N} \mapsto \mathbb{R}$ we define the $\mathcal{A}$-quasiconvexification of $f$ at $\Sigma$ as

$$
\mathcal{Q}_{\mathcal{A}} f(\Sigma):=\inf \left\{\int_{Q} f(\Sigma+V(x)) \mathrm{d} x: V \in C_{\#}^{\infty}\left(\mathbb{R}^{N} ; \mathbb{M}^{d \times N}\right) \cap \operatorname{Ker} \mathcal{A}, \int_{Q} V(y) \mathrm{d} y=0\right\} .
$$

Remark 2.3. If $f(x, \cdot)$ is upper semicontinuous for a.e. $x \in \Omega$ then $\mathcal{Q}_{\mathcal{A}} f(x, \cdot)$ is $\mathcal{A}$-quasiconvex (see [15], Prop. 3.4). Moreover, if $f$ is upper semicontinuous and locally bounded from above, then, by Fatou's lemma, we may replace $C^{\infty}\left(\mathbb{R}^{N} ; \mathbb{M}^{d \times N}\right)$ by $L^{\infty}\left(\mathbb{R}^{N} ; \mathbb{M}^{d \times N}\right)$ in Definitions 2.1 and 2.2. If, in addition, $|f(\Sigma)| \leq \beta\left(1+|\Sigma|^{p}\right)$ for some $\beta>0$ and for every $\Sigma \in \mathbb{M}^{d \times N}$, then $C^{\infty}\left(\mathbb{R}^{N} ; \mathbb{M}^{d \times N}\right)$ may be replaced by $L^{p}\left(\mathbb{R}^{N} ; \mathbb{M}^{d \times N}\right.$ ) (see $[15]$, Rem. 3.3(ii)).

In [15] Fonseca and Müller prove that the $\mathcal{A}$-quasiconvexity is a necessary and sufficient condition for the lower semicontinuity of integral functionals of the form

$$
F(V)=\int_{\Omega} f(x, V(x)) \mathrm{d} x
$$

under the PDE constraint $\mathcal{A} V=0$ and $f$ Carathéodory (see [15], Thms. 3.6 and 3.7).

The following proposition turns out to be an important tool to prove Theorem 4.1.

Proposition 2.4 ([15], Prop. 3.9). Let $\left(V_{n}\right) \subset L^{\infty}\left(\Omega ; \mathbb{M}^{d \times N}\right) \cap \operatorname{Ker} \mathcal{A}$ be a sequence such that $V_{n} \rightarrow V$ weakly* in $L^{\infty}\left(\Omega ; \mathbb{M}^{d \times N}\right)$ and such that $\left(V_{n}\right)$ generates a Young measure $\mu$. Then there exists a neglibible set $N \subset \Omega$ such that for every $x_{0} \in \Omega \backslash N$ and for every subcube $Q^{\prime} \subset \subset Q$ there exists a sequence $\left(\bar{W}_{n}\right) \subset L_{\#}^{\infty}\left(Q ; \mathbb{M}^{d \times N}\right) \cap \operatorname{Ker} \mathcal{A}$ such that

1. $\bar{W}_{n} \rightarrow V\left(x_{0}\right)$ weakly* in $L^{\infty}$;

2. $\int_{Q} \bar{W}_{n}(y) \mathrm{d} y=V\left(x_{0}\right)$;

3. $\bar{W}_{n}$ generates a Young measure $\nu$ such that for every $f \in C_{c}\left(\mathbb{M}^{d \times N}\right)$

$$
\left|\int_{Q}\left\langle\nu_{y}, f\right\rangle \mathrm{d} y-\left\langle\mu_{x_{0}}, f\right\rangle\right| \leq \mathcal{L}^{N}\left(Q \backslash Q^{\prime}\right)\|f\|_{L^{\infty}(B(0,3 K))}
$$

where $K=\sup _{n}\left\|V_{n}\right\|_{\infty}$. In addition, if $f: \mathbb{M}^{d \times N} \rightarrow \mathbb{R}$ is continuous and $\mathcal{A}$-quasiconvex then

$$
f\left(V\left(x_{0}\right)\right) \leq\left\langle\mu_{x_{0}}, f\right\rangle
$$

for every $x_{0} \in \Omega \backslash N$. 
Remark 2.5. Let $\mu, \nu, x_{0} \in \Omega \backslash N$ and $Q^{\prime} \subset \subset Q$ be as in Proposition 2.4. If $f: \mathbb{M}^{d \times N} \rightarrow[0,+\infty)$ is locally bounded and lower semicontinuous then the inequality

$$
\int_{Q}\left\langle\nu_{y}, f\right\rangle \mathrm{d} y \leq\left\langle\mu_{x_{0}}, f\right\rangle+\mathcal{L}^{N}\left(Q \backslash Q^{\prime}\right)\|f\|_{L^{\infty}(B(0,3 K))}
$$

still holds true.

We first prove (2.11) for every continuous and nonnegative function $f$. Let $\varphi \in C_{c}^{1}\left(\mathbb{M}^{d \times N}\right)$ be a cut-off function such that

$$
\varphi(\Sigma):=\left\{\begin{array}{l}
1 \text { if }|\Sigma| \leq 1 \\
0 \text { if }|\Sigma| \geq 2
\end{array}\right.
$$

We define $f_{n}(\Sigma):=\varphi(\Sigma / n) f(\Sigma)$. Then, $f_{n} \in C_{c}\left(\mathbb{M}^{d \times N}\right)$ and it satisfies

$$
\left\langle\nu_{y}, f_{n}\right\rangle=\int_{\mathbb{M}^{d \times N}} f_{n}(\Sigma) \mathrm{d} \nu_{y}(\Sigma) \geq \int_{B_{n}(0)} f(\Sigma) \mathrm{d} \nu_{y}(\Sigma)
$$

for every $y \in Q$. This implies that

$$
\liminf _{n \rightarrow \infty}\left\langle\nu_{y}, f_{n}\right\rangle \geq \int_{\mathbb{M}^{d \times N}} f(\Sigma) \mathrm{d} \nu_{y}(\Sigma)
$$

for every $y \in Q$. By Fatou's Lemma and (2.9) with $f_{n}$ in place of $f$, we have that

$$
\begin{aligned}
\int_{Q}\left\langle\nu_{y}, f\right\rangle \mathrm{d} y & \leq \liminf _{n \rightarrow \infty} \int_{Q}\left\langle\nu_{y}, f_{n}\right\rangle \mathrm{d} y \\
& \leq \liminf _{n \rightarrow \infty}\left\langle\mu_{x_{0}}, f_{n}\right\rangle+\mathcal{L}^{N}\left(Q \backslash Q^{\prime}\right)\left\|f_{n}\right\|_{L^{\infty}(B(0,3 K))} \\
& \leq\left\langle\mu_{x_{0}}, f\right\rangle+\mathcal{L}^{N}\left(Q \backslash Q^{\prime}\right)\|f\|_{L^{\infty}(B(0,3 K))},
\end{aligned}
$$

which proves (2.11) when $f$ is continuous and nonnegative.

We now assume that $f: \mathbb{M}^{d \times N} \rightarrow[0,+\infty)$ is a locally bounded and lower semicontinuous function. Then, $f=\sup _{\lambda>0} f_{\lambda}$ where $f_{\lambda}$ is its Yosida transform for every $\lambda>0$ (see e.g. [10]). Since $f_{\lambda}$ is continuous and nonnegative, by (2.13) we find that

$$
\int_{Q}\left\langle\nu_{y}, f_{\lambda}\right\rangle \mathrm{d} y \leq\left\langle\mu_{x_{0}}, f_{\lambda}\right\rangle+\mathcal{L}^{N}\left(Q \backslash Q^{\prime}\right)\left\|f_{\lambda}\right\|_{L^{\infty}(B(0,3 K))}, \quad \forall \lambda>0 .
$$

Moreover, $0 \leq f_{\lambda} \leq f$; hence, we get that

$$
\left\|f_{\lambda}\right\|_{L^{\infty}(B(0,3 K))} \leq\|f\|_{L^{\infty}(B(0,3 K))}, \quad \forall \lambda>0 .
$$

By Beppo-Levi's Theorem, we can pass to the limit in (2.14), as $\lambda \rightarrow 0^{+}$, and the inequality (2.11) holds true.

Remark 2.6. Let $\mu=\left(\mu_{x}\right)_{x \in \Omega}$ be a $\mathcal{A}$ - $\infty$-Young measure. Then, reasoning as in Proposition 2.4, we can easily check that for every continuous functions $f$ the following inequality

$$
\mathcal{Q}_{\mathcal{A}} f\left(\int_{\mathbb{M}^{d \times N}} \Sigma \mathrm{d} \mu_{x}(\Sigma)\right) \leq\left\langle\mu_{x}, f\right\rangle,
$$

holds true for a.e. $x \in \Omega$. 


\section{2. $\mathcal{A}-\infty$ and $\mathcal{A}$-weak quasiconvexity}

In this section we recall some definitions and results contained in ([3], Sect. 3). We also prove Proposition 2.9 which improves ([19], Cor. 3.10) and ([3], Prop. 3.6(iv)). As a consequence, in Theorem 2.10 we prove an $L^{p}$-approximation result by way of $\Gamma$-convergence.

Definition 2.7. We say that a Borel function $f: \mathbb{M}^{d \times N} \mapsto[0,+\infty)$ is $\mathcal{A}$ - $\infty$ quasiconvex if for every $\Sigma \in \mathbb{M}^{d \times N}$

$$
f(\Sigma)=\lim _{p \rightarrow \infty} \inf \left\{\left(\int_{Q} f^{p}(\Sigma+V(x)) \mathrm{d} x\right)^{1 / p}: V \in L_{\#}^{\infty}\left(Q ; \mathbb{M}^{d \times N}\right) \cap \operatorname{Ker} \mathcal{A}, \int_{Q} V \mathrm{~d} x=0\right\} .
$$

Here and in the sequel we denote by $L_{\#}^{\infty}\left(Q ; \mathbb{M}^{d \times N}\right)$ the set of $L_{\mathrm{loc}}^{\infty}\left(\mathbb{R}^{N} ; \mathbb{M}^{d \times N}\right)$ functions which are $Q$-periodic and we define

$$
f_{p}(\Sigma):=\inf \left\{\left(\int_{Q} f^{p}(\Sigma+V(x)) \mathrm{d} x\right)^{1 / p}: V \in L_{\#}^{\infty}\left(Q ; \mathbb{M}^{d \times N}\right) \cap \operatorname{Ker} \mathcal{A}, \int_{Q} V \mathrm{~d} x=0\right\} .
$$

For any function $f: \mathbb{M}^{d \times N} \rightarrow \mathbb{R}$ we define the $\mathcal{A}-\infty$ quasiconvex envelope of $f$ as

$$
\mathcal{Q}_{\mathcal{A}}^{\infty} f(\Sigma):=\sup \{h(\Sigma): h \text { is } \mathcal{A}-\infty \text { quasiconvex and } h \leq f\} .
$$

In ([3], Prop. 3.8) we prove that if $f$ is a continuous function, then $f_{p}$ is an $\mathcal{A}$-quasiconvex function and $\mathcal{Q}_{\mathcal{A}}^{\infty} f$ is an $\mathcal{A}$ - $\infty$ quasiconvex function. The $\mathcal{A}$ - $\infty$ quasiconvex envelope of $f$ can be also obtained as limit of $f_{p}$ as $p$ tends to $+\infty$; i.e.,

$$
\mathcal{Q}_{\mathcal{A}}^{\infty} f(\Sigma)=\lim _{p \rightarrow \infty} \inf \left\{\left(\int_{Q} f^{p}(\Sigma+V(y)) \mathrm{d} y\right)^{1 / p}: V \in L_{\#}^{\infty}\left(Q ; \mathbb{M}^{d \times N}\right) \cap \operatorname{Ker} \mathcal{A}, \int_{Q} V \mathrm{~d} y=0\right\},
$$

for every $\Sigma \in \mathbb{M}^{d \times N}$. In ([3], Thm. 4.2) we prove that the $\Gamma$-limit of a sequence of power-law functionals, as in (1.4), is given by the supremal functional

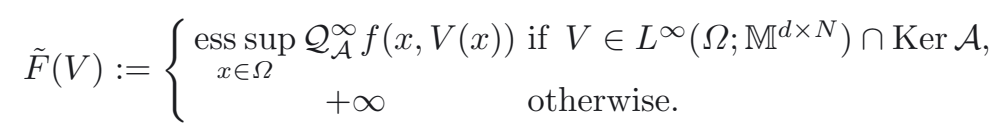

In ([3], Def. 3.1) we also introduce the notion of $\mathcal{A}$-weak quasiconvex functions.

Definition 2.8. We say that a Borel function $f: \mathbb{M}^{d \times N} \mapsto \mathbb{R}$ is $\mathcal{A}$-weak quasiconvex if for all $\Sigma \in \mathbb{M}^{d \times N}$

$$
f(\Sigma) \leq \operatorname{ess}_{x \in Q} f(\Sigma+V(x))
$$

for every $V \in L_{\#}^{\infty}\left(Q ; \mathbb{M}^{d \times N}\right) \cap \operatorname{Ker} \mathcal{A}$ with $\int_{Q} V \mathrm{~d} x=0$.

In ([3], Prop. 3.6) we prove that

$\mathcal{A}$-quasiconvexity $\Longrightarrow \mathcal{A}$ - $\infty$ quasiconvexity $\Longrightarrow \mathcal{A}$-weak quasiconvexity;

while, the counter implications are false (see [3], Sect. 5.2). In ([3], Prop. 3.6) we also prove that

if $f$ is lower semicontinuous and level convex $\Longrightarrow f$ is $\mathcal{A}$-weak quasiconvex.

We recall that a function $f: \mathbb{M}^{d \times N} \rightarrow \mathbb{R}$ is level convex if

$$
f\left(t \Sigma_{1}+(1-t) \Sigma_{2}\right) \leq f\left(\Sigma_{1}\right) \vee f\left(\Sigma_{2}\right), \quad \forall \Sigma_{1}, \Sigma_{2} \in \mathbb{M}^{d \times N}, \forall t \in[0,1] .
$$

Finally, in the following proposition we prove that

if $f$ is lower semicontinuous, coercive and level convex $\Longrightarrow f$ is $\mathcal{A}-\infty$ quasiconvex. 
Proposition 2.9. Let $f: \mathbb{R}^{N} \rightarrow \mathbb{R}$ be a level convex, lower semicontinuous function satisfying

$$
f(\xi) \geq \alpha|\xi|
$$

for a fixed $\alpha>0$ and for every $\xi \in \mathbb{R}^{N}$. Let $\left(f^{p}\right)^{* *}$ be the convex envelope of $f^{p}$ for every $p \geq 1$. Then

$$
\lim _{p \rightarrow \infty}\left(\left(f^{p}\right)^{* *}\right)^{1 / p}(\xi)=f(\xi)
$$

for every $\xi \in \mathbb{R}^{N}$. In particular, $f$ is an $\mathcal{A}-\infty$ quasiconvex function.

Proof. We can easily prove that $\left(\left(\left(f^{p}\right)^{* *}\right)^{1 / p}\right)$ is a nondecreasing sequence. Since $f$ is lower semicontinuous we have that $f=\sup _{\lambda>0} f_{\lambda}$ where $f_{\lambda}$ is the $\lambda$-Lipschitz continuous function given by

$$
f_{\lambda}(\xi)=\inf \left\{f(\eta) \vee \lambda|\xi-\eta|: \eta \in \mathbb{R}^{N}\right\}
$$

(see [1], Props. 2.5 and 2.6). Note that $f_{\lambda}$ is also coercive; i.e., $f_{\lambda}(\xi) \geq \alpha|\xi|$ for every $\lambda>0$ and for every $\xi \in \mathbb{R}^{N}$. In addition, since $f$ is level convex, then $f_{\lambda}$ is also level convex for every $\lambda>0$. In fact, let $\xi_{1}, \xi_{2} \in \mathbb{R}^{N}$. For every fixed $\varepsilon>0$ let $\eta_{1}, \eta_{2} \in \mathbb{R}^{N}$ be such that

$$
f_{\lambda}\left(\xi_{1}\right) \geq f\left(\eta_{1}\right) \vee \lambda\left|\xi_{1}-\eta_{1}\right|-\varepsilon, \quad f_{\lambda}\left(\xi_{2}\right) \geq f\left(\eta_{2}\right) \vee \lambda\left|\xi_{2}-\eta_{2}\right|-\varepsilon .
$$

Then, for every $t \in(0,1)$, we find that

$$
\begin{aligned}
f_{\lambda}\left(t \xi_{1}+(1-t) \xi_{2}\right) & \leq f\left(t \eta_{1}+(1-t) \eta_{2}\right) \vee \lambda\left|t\left(\xi_{1}-\eta_{1}\right)+(1-t)\left(\xi_{2}-\eta_{2}\right)\right| \\
& \leq f\left(\eta_{1}\right) \vee g\left(\eta_{2}\right) \vee \lambda\left|\xi_{1}-\eta_{1}\right| \vee \lambda\left|\xi_{2}-\eta_{2}\right| \\
& \leq f_{\lambda}\left(\xi_{1}\right) \vee g_{\lambda}\left(\xi_{2}\right)+\varepsilon .
\end{aligned}
$$

By the arbitrariness of $\varepsilon$, it follows that

$$
f_{\lambda}\left(\theta \xi_{1}+(1-\theta) \xi_{2}\right) \leq f_{\lambda}\left(\xi_{1}\right) \vee f_{\lambda}\left(\xi_{2}\right)
$$

that is, $f_{\lambda}$ is a level convex function.

By [19], Corollary 3.10, we find that

$$
\sup _{p>1}\left(\left(f_{\lambda}^{p}\right)^{* *}\right)^{1 / p}(\xi)=f_{\lambda}(\xi)
$$

for every $\xi \in \mathbb{R}^{N}$. This implies that

$$
f(\xi)=\sup _{\lambda>0} f_{\lambda}(\xi)=\sup _{\lambda>0}\left(\sup _{p>1}\left(\left(f_{\lambda}^{p}\right)^{* *}\right)^{1 / p}(\xi)\right)=\sup _{p>1}\left(\sup _{\lambda>0}\left(\left(f_{\lambda}^{p}\right)^{* *}\right)^{1 / p}(\xi)\right) \leq \sup _{p>1}\left(\left(f^{p}\right)^{* *}\right)^{1 / p}(\xi) \leq f(\xi),
$$

which proves formula $(2.21)$.

We now demonstrate that $f$ is an $\mathcal{A}-\infty$ quasiconvex function. By Jensen's inequality we have that

$$
\left(f^{p}\right)^{* *}(\Sigma) \leq \int_{Q}\left(f^{p}\right)^{* *}(\Sigma+V(x)) \mathrm{d} x \leq \int_{Q} f^{p}(\Sigma+V(x)) \mathrm{d} x
$$

for every $V \in L_{\#}^{\infty}\left(Q ; \mathbb{M}^{d \times N}\right) \cap \operatorname{Ker} \mathcal{A}$ such that $\int_{Q} V \mathrm{~d} x=0$. It follows that

$$
\left(f^{p}\right)^{* *}(\Sigma) \leq \inf \left\{\int_{Q} f^{p}(\Sigma+V(x)) \mathrm{d} x: V \in L_{\#}^{\infty}\left(Q ; \mathbb{M}^{d \times N}\right) \cap \operatorname{Ker} \mathcal{A}, \int_{Q} V \mathrm{~d} x=0\right\}=f_{p}^{p}(\Sigma) ;
$$

hence, $\left(\left(f^{p}\right)^{* *}\right)^{1 / p} \leq f_{p} \leq f$. Passing to the limit as $p \rightarrow \infty$ we get, in particular, that $f=\lim _{p \rightarrow \infty} f_{p}$ which concludes the proof.

We now prove the following $\Gamma$-convergence result for power-law functionals. 
Theorem 2.10. Let $f: \Omega \times \mathbb{M}^{d \times N} \mapsto \mathbb{R}$ be a Borel function such that $f(x, \cdot)$ is lower semicontinuous and level convex for a.e. $x \in \Omega$ and satisfies the growth condition: there exists $\alpha>0$ such that

$$
f(x, \Sigma) \geq \alpha|\Sigma| \quad \text { for a.e } x \in \Omega, \text { for every } \Sigma \in \mathbb{M}^{d \times N} .
$$

Let $F_{p}, F: L^{1}\left(\Omega ; \mathbb{M}^{d \times N}\right) \rightarrow \mathbb{R} \cup\{+\infty\}$ be given by

$$
F_{p}(V):=\left\{\begin{array}{cl}
\left(\int_{\Omega} f^{p}(x, V(x)) \mathrm{d} x\right)^{1 / p} & \text { if } V \in L^{p}\left(\Omega ; \mathbb{M}^{d \times N}\right) \cap \operatorname{Ker} \mathcal{A} \\
+\infty & \text { otherwise }
\end{array}\right.
$$

and

$$
F(V):=\left\{\begin{array}{cl}
\operatorname{esssup}_{x \in \Omega} f(x, V(x)) & \text { if } V \in L^{\infty}\left(\Omega ; \mathbb{M}^{d \times N}\right) \cap \operatorname{Ker} \mathcal{A} \\
+\infty & \text { otherwise, }
\end{array}\right.
$$

respectively. Then

$$
\Gamma\left(w-L^{1}\right)-\lim _{p \rightarrow \infty} F_{p}(V)=F(V)
$$

where $\Gamma\left(w-L^{1}\right)$ denotes the $\Gamma$-limit with respect to the $L^{1}$-weak topology.

Proof. We remark that $\left(|\Omega|^{-1 / p} F_{p}\right)$ is a nondecreasing sequence and it converges pointwise to the functional $F$; hence, by ([14], Props $6.7,5.1)$, we have that

$$
\Gamma\left(w-L^{1}\right)-\lim _{p \rightarrow \infty} F_{p}(V) \leq F(V) .
$$

Conversely, since $\left(f^{p}\right)^{* *}(x, \cdot)$ is a convex function we have that the functional $G_{p}: L^{1}\left(\Omega ; \mathbb{R}^{N}\right) \rightarrow \mathbb{R} \cup\{+\infty\}$ given by

$$
G_{p}(V)=\left\{\begin{array}{cl}
\left(\int_{\Omega}\left(f^{p}\right)^{* *}(x, V(x)) \mathrm{d} x\right)^{1 / p} & \text { if } V \in L^{p}\left(\Omega, \mathbb{M}^{d \times N}\right) \cap \operatorname{Ker} \mathcal{A} \\
+\infty & \text { otherwise }
\end{array}\right.
$$

is lower semicontinuous with respect to the $L^{1}$-weak topology. Moreover, $G_{p} \leq F_{p}$ for every $p \geq 1$. Therefore, by ([14], Prop. 5.4), we find that

$$
\Gamma\left(w-L^{1}\right)-\lim _{p \rightarrow \infty} F_{p}(V) \geq \Gamma\left(w-L^{1}\right)-\lim _{p \rightarrow \infty} G_{p}(V)=\sup _{p>1} G_{p}(V) .
$$

By Proposition 2.9 and reasoning as in the proof of ([3], Thm. 4.2), we easily get that

$$
\sup _{p>1} G_{p}(V)=F(V) \text {. }
$$

Therefore, we conclude that $\Gamma\left(w-L^{1}\right)-\lim _{p \rightarrow \infty} F_{p}(V)=F(V)$.

\section{3. $\mathcal{A}$-Young QuASICONVEX FUnCtions}

In order to study the $L^{\infty}$-weak* lower semicontinuity of supremal functionals as in (1.1) we introduce the following class of functions.

Definition 3.1. We say that a Borel function $f: \mathbb{M}^{d \times N} \mapsto \mathbb{R}$ is $\mathcal{A}$-Young quasiconvex if for every $\mathcal{A}$ - $\infty$-Young measure $\mu=\left(\mu_{x}\right)_{x \in Q}$ it satisfies the following Jensen's inequality for Young measures

$$
f\left(\int_{\mathbb{M}^{d \times N}} \Sigma \mathrm{d} \mu_{x}(\Sigma)\right) \leq \underset{x \in Q}{\operatorname{ess} \sup } \mu_{x^{-}} \operatorname{ess~sup}_{\Sigma \in \mathbb{M}^{d \times N}} f(\Sigma)
$$

for a.e. $x \in Q$. 
We remark that the class of $\mathcal{A}$-Young quasiconvex functions is quite large. In fact, in the following Propositions 3.3 and 3.4, under suitable assumptions, we prove that this class contains strictly the more relevant classes of functions as $\mathcal{A}$-quasiconvex functions, level convex functions, $\mathcal{A}$ - $\infty$ quasiconvex functions and $\mathcal{A}-\infty$ quasiconvex envelope functions (see also Rem. 3.5).

In order to prove Proposition 3.3(2) we recall the Jensen's inequality introduced by Barron et al. in [6].

Theorem 3.2 ([7], Thm. 1.2). Let $f: \mathbb{R}^{k} \rightarrow \mathbb{R}$ be a lower semicontinuous and level convex function, and let $\mu$ be a probability measure supported on $\Omega$. Then, for every function $u \in L_{\mu}^{1}\left(\Omega ; \mathbb{R}^{k}\right)$, we have

$$
f\left(\int_{\Omega} u(x) \mathrm{d} \mu(x)\right) \leq \mu \text { - } \underset{x \in \Omega}{\operatorname{ess} \sup }(f \circ u)(x) .
$$

Proposition 3.3. Let $f: \mathbb{M}^{d \times N} \mapsto \mathbb{R}$.

(1) If $f$ is a continuous and $\mathcal{A}$-quasiconvex function, then $f$ is an $\mathcal{A}$-Young quasiconvex function;

(2) If $f$ is a lower semicontinuous and level convex function, then $f$ is an $\mathcal{A}$-Young quasiconvex function.

Proof.

(1) By Proposition 2.4, we find that for every $\mathcal{A}$ - $\infty$-Young measure $\mu=\left(\mu_{x}\right)_{x \in Q}$

$$
f\left(\int_{\mathbb{M}^{d \times N}} \Sigma \mathrm{d} \mu_{x}(\Sigma)\right) \leq \int_{\mathbb{M}^{d \times N}} f(\Sigma) \mathrm{d} \mu_{x}(\Sigma)
$$

for a.e. $x \in Q$. Therefore, $f$ satisfies (3.25).

(2) By the Fundamental Theorem on Young Measure (see e.g. [15], Thm. 2.2(5)) we have that every $\mathcal{A}$ - $\infty$-Young measure is a probability measure. Therefore, by Theorem 3.2, we easily get that any lower semicontinuous and level convex function is, in particular, an $\mathcal{A}$-Young quasiconvex function.

Proposition 3.4. Let $f: \mathbb{M}^{d \times N} \mapsto[0,+\infty)$ be a continuous function.

(1) If $f$ is an $\mathcal{A}-\infty$ quasiconvex function, then for every open set $\Omega \subset \mathbb{R}^{N}$ and for every $\mathcal{A}-\infty$-Young measure $\mu=\left(\mu_{x}\right)_{x \in \Omega}$ we have that

$$
f\left(\int_{\mathbb{M}^{d \times N}} \Sigma \mathrm{d} \mu_{x}(\Sigma)\right) \leq \mu_{x}-\underset{\Sigma \in \mathbb{M}^{d \times N}}{\operatorname{ess} \sup } f(\Sigma)
$$

for a.e. $x \in \Omega$. In particular, $f$ is an $\mathcal{A}$-Young quasiconvex function.

(2) If $f$ satisfies the growth condition $f(\Sigma) \geq \alpha|\Sigma|$, for $\alpha>0$ and for every $\Sigma \in \mathbb{M}^{d \times N}$, then $\mathcal{Q}_{\mathcal{A}}^{\infty} f$ is a lower semicontinuous and $\mathcal{A}$-Young quasiconvex function.

Proof.

(1) Let $\mu$ be an $\mathcal{A}$ - $\infty$-Young measure generated by a sequence $\left(V_{h}\right) \in L^{\infty}\left(\Omega ; \mathbb{M}^{d \times N}\right) \cap \operatorname{Ker} \mathcal{A}$ weakly* converging to $V \in L^{\infty}\left(\Omega ; \mathbb{M}^{d \times N}\right)$. In particular, we have that

$$
V(x)=\int_{\mathbb{M}^{d \times N}} \Sigma \mathrm{d} \mu_{x}(\Sigma),
$$

for a.e. $x \in \Omega$ (see e.g. [15], Rem. 2.3(ii)). By Remark 2.6, it follows that

$$
\mathcal{Q}_{\mathcal{A}} f^{p}(V(x)) \leq \mu_{x^{-}} \operatorname{esssup}_{\Sigma \in \mathbb{M}^{d \times N}} f^{p}(\Sigma)
$$


for a.e. $x \in \Omega$. Since, by definition, $f_{p}^{p} \leq \mathcal{Q}_{\mathcal{A}} f^{p}$ for every $p \geq 1$, we get that

$$
f_{p}\left(\int_{\mathbb{M}^{d \times N}} \Sigma \mathrm{d} \mu_{x}(\Sigma)\right) \leq \mu_{x^{-}} \underset{\Sigma \in \mathbb{M}^{d \times N}}{\operatorname{ess} \sup } f(\Sigma) .
$$

Passing to the limit as $p \rightarrow \infty$, we obtain

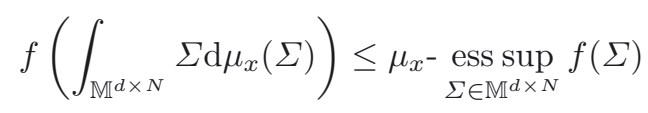

for a.e. $x \in \Omega$. By Definition 3.1 we get that $f$ is also an $\mathcal{A}$-Young quasiconvex function.

(2) By ([3], Props. 3.8 and 3.9) we have that $\left(f_{p}^{p}\right)$ is a sequence of continuous and $\mathcal{A}$-quasiconvex functions and $\sup _{p} f_{p}=\mathcal{Q}_{\mathcal{A}}^{\infty} f$. In particular, $\mathcal{Q}_{\mathcal{A}}^{\infty} f$ is a lower semicontinuous function. With a slight abuse of notation, we denote by $f_{n}$ the function given by $f_{p}$ with $p=1 / n$.

By Proposition 2.4, we have that for every fixed $\mathcal{A}$ - $\infty$-Young measure $\mu=\left(\mu_{x}\right)_{x \in \Omega}$ and for every fixed $n \in \mathbb{N}$, there exists a negligible set $H_{n} \subset \Omega$ such that

$$
f_{n}\left(\int_{\mathbb{M}^{d \times N}} \Sigma \mathrm{d} \mu_{x}(\Sigma)\right)=f_{n}(V(x)) \leq\left\langle\mu_{x}, f_{n}^{n}\right\rangle^{1 / n} \leq \mu_{x^{-}} \underset{\Sigma \in \mathbb{M}^{d \times N}}{\operatorname{ess} \sup _{\mathcal{A}}} \mathcal{Q}_{\mathcal{A}}^{\infty} f(\Sigma)
$$

for every $x \in \Omega \backslash H_{n}$. Set $H=\bigcup_{n} H_{n}$. Then, we find that

$$
f_{n}\left(\int_{\mathbb{M}^{d \times N}} \Sigma \mathrm{d} \mu_{x}(\Sigma)\right) \leq \mu_{x^{-}} \underset{\Sigma \in \mathbb{M}^{d \times N}}{\operatorname{ess} \sup _{\mathcal{A}}} \mathcal{Q}_{\mathcal{A}}^{\infty} f(\Sigma)
$$

for every $x \in \Omega \backslash H$. Hence, by ([3], Prop. 3.8), passing to the limit as $n \rightarrow \infty$, we have that

$$
Q_{\mathcal{A}}^{\infty} f\left(\int_{\mathbb{M}^{d \times N}} \Sigma \mathrm{d} \mu_{x}(\Sigma)\right) \leq \mu_{x^{-}} \operatorname{ess}_{\Sigma \in \mathbb{M}^{d \times N}} \mathcal{Q}_{\mathcal{A}}^{\infty} f(\Sigma)
$$

for every $x \in \Omega \backslash H$. In particular, if $\Omega=Q$ we obtain that $Q_{\mathcal{A}}^{\infty} f$ is an $\mathcal{A}$-Young quasiconvex function.

\section{Remark 3.5.}

(1) Note that, by ([3], Thm. 4.2) and Proposition 3.4(2), if $f$ is a Carathéodory function, then the $\Gamma$-limit of $\left(F_{p}\right)$, as in (1.4), is a supremal functional whose supremand function is an $\mathcal{A}$-Young quasiconvex function with respect to the second variable.

(2) In Section 6.1 we exhibit an example which shows that if $d, N>1$, then

$$
\mathcal{A} \text {-Young quasiconvexity } \Longrightarrow\left\{\begin{array}{l}
\mathcal{A} \text {-quasiconvexity } \\
\text { level convexity } \\
\mathcal{A}-\infty \text { quasiconvexity }
\end{array}\right.
$$

(see Example 6.7).

\section{A sufficient CONDition For the $w^{*}$-LOWER SEMiCOntinuity}

In this section we deal with the lower semicontinuity of a supremal functionals $F$ under a differential constraint defined by

$$
F(V):=\operatorname{ess}_{x \in \Omega} f(x, V(x)), \quad V \in L^{\infty}\left(\Omega ; \mathbb{M}^{d \times N}\right) \cap \operatorname{Ker} \mathcal{A} .
$$


In Theorem 4.1 we prove the lower semicontinuity of $F$ under the hypotheses that $f$ is a coercive, lower semicontinuous and $\mathcal{A}$-Young quasiconvex function in the second variable. In Theorem 4.2 we remove the coercivity assumption. Note that, if $\mathcal{A}=$ curl, then Theorem 4.2 generalises ([13], Thm. 3.4).

It is still an open question whether the $\mathcal{A}$-Young quasiconvexity is also a necessary condition for the lower semicontinuity of $F$ when $d, N>1$.

Theorem 4.1. Let $f: \Omega \times \mathbb{M}^{d \times N} \rightarrow[0,+\infty)$ be a Borel function such that $f(x, \cdot)$ is lower semicontinuous and locally bounded for a.e. $x \in \Omega$. Assume that

(i) $f$ satisfies the growth condition: there exists $\alpha>0$ such that

$$
f(x, \Sigma) \geq \alpha|\Sigma| \quad \text { for a.e } x \in \Omega, \text { for every } \Sigma \in \mathbb{M}^{d \times N} ;
$$

(ii) $f(x, \cdot)$ is $\mathcal{A}$-Young quasiconvex for a.e. $x \in \Omega$.

Let $F, F_{p}: L^{\infty}\left(\Omega ; \mathbb{M}^{d \times N}\right) \rightarrow \mathbb{R} \cup\{+\infty\}$ be the functionals defined by (1.3) and (1.4), respectively.

Then, for every $V,\left(V_{p}\right) \subset L^{\infty}\left(\Omega ; \mathbb{M}^{d \times N}\right)$ such that $V_{p} \rightarrow V$ weakly* in $L^{\infty}\left(\Omega ; \mathbb{M}^{d \times N}\right)$, we have

$$
F(V) \leq \liminf _{p \rightarrow \infty} F_{p}\left(V_{p}\right) .
$$

In particular the functional $F$ is sequentially lower semicontinuous with respect to the $L^{\infty}$ - weak* convergence.

Proof. Let $\left(V_{p}\right) \in L^{\infty}\left(\Omega ; \mathbb{M}^{d \times N}\right)$ be a sequence $L^{\infty}$-weakly* converging to $V \in L^{\infty}\left(\Omega ; \mathbb{M}^{d \times N}\right)$. We may always assume that $\liminf _{p \rightarrow \infty} F_{p}\left(V_{p}\right)<+\infty$. In particular, $V_{p} \in L^{\infty}\left(\Omega ; \mathbb{M}^{d \times N}\right) \cap$ Ker $\mathcal{A}$; hence, also $V \in L^{\infty}\left(\Omega ; \mathbb{M}^{d \times N}\right) \cap \operatorname{Ker} \mathcal{A}$. In fact,

$$
\langle\mathcal{A} V, \phi\rangle=\lim _{p \rightarrow+\infty}\left\langle\mathcal{A} V_{p}, \phi\right\rangle=0, \quad \forall \phi \in C_{0}^{\infty}\left(\Omega, \mathbb{M}^{d \times N}\right) .
$$

By the density of the subset $C_{0}^{\infty}\left(\Omega, \mathbb{M}^{d \times N}\right)$ in $W_{0}^{1,1}\left(\Omega, \mathbb{M}^{d \times N}\right)$, with respect to the strong convergence, we get that also $V$ satisfies the constraint $\mathcal{A} V=0$.

The sequence $\left(V_{p}\right)$ generates a Young measure $\left(\mu_{x}\right)_{x \in \Omega}$ such that

$$
V(x)=\int_{\mathbb{R}^{d \times N}} \Sigma \mathrm{d} \mu_{x}(\Sigma)
$$

for a.e. $x \in \Omega$. In particular, by the Fundamental Theorem on Young Measure (see e.g. [15], Thm. 2.2(5)) for any fixed $q>1$, we have that

$$
\begin{aligned}
\liminf _{p \rightarrow \infty} F_{q}\left(V_{p}\right) & =\liminf _{p \rightarrow \infty}\left(\int_{\Omega} f^{q}\left(x, V_{p}(x)\right) \mathrm{d} x\right)^{1 / q} \\
& \geq\left(\int_{\Omega} \int_{\mathbb{M}^{d \times N}} f^{q}(x, \Sigma) \mathrm{d} \mu_{x}(\Sigma) \mathrm{d} x\right)^{1 / q} .
\end{aligned}
$$

By applying ([3], Lem. 4.5) we obtain

$$
\liminf _{q \rightarrow \infty} \liminf _{p \rightarrow \infty} F_{q}\left(V_{p}\right) \geq \underset{x \in \Omega}{\operatorname{ess} \sup }\left(\mu_{x^{-}} \operatorname{ess~sup}_{\Sigma \in \mathbb{M}^{d \times N}} f(x, \Sigma)\right) .
$$

By (4.29), we get that

$$
\begin{aligned}
\underset{x \in \Omega}{\operatorname{ess} \sup }\left(\mu_{x^{-}} \underset{\Sigma \in \mathbb{M}^{d \times N}}{\operatorname{ess} \sup } f(x, \Sigma)\right) & \leq \liminf _{q \rightarrow \infty} \liminf _{p \rightarrow \infty} F_{q}\left(V_{p}\right) \\
& \leq \liminf _{q \rightarrow \infty} \liminf _{p \rightarrow \infty}|\Omega|^{1 / q-1 / p} F_{p}\left(V_{p}\right) \\
& =\liminf _{p \rightarrow \infty} F_{p}\left(V_{p}\right) .
\end{aligned}
$$


If we prove that

$$
f\left(x_{0}, V\left(x_{0}\right)\right) \leq \operatorname{ess~sup}_{x \in \Omega}\left(\mu_{x^{-}} \operatorname{ess~sup}_{\Sigma \in \mathbb{M}^{d \times N}} f(x, \Sigma)\right)
$$

for a.e. $x_{0} \in \Omega$, by (4.30), we infer that

$$
F(V) \leq \liminf _{p \rightarrow \infty} F_{p}\left(V_{p}\right)
$$

By Proposition 2.4 and Remark 2.5, there exists a negligible set $N \subset \Omega$ such that for every $x_{0} \in \Omega \backslash N$ and for every $Q^{\prime} \subset \subset Q$ we can construct a sequence $\left(\bar{W}_{n}\right) \subset L_{\#}^{\infty}\left(Q ; \mathbb{M}^{d \times N}\right) \cap \operatorname{Ker} \mathcal{A}$ satisfying the following properties

(1) $\bar{W}_{n} \rightarrow V\left(x_{0}\right)$ weakly* in $L^{\infty}\left(\Omega ; \mathbb{M}^{d \times N}\right)$;

(2) $\int_{Q} \bar{W}_{n}(x) \mathrm{d} x=V\left(x_{0}\right)$;

(3) $\left(\bar{W}_{n}\right)$ generates a Young measure $\nu^{x_{0}}$ such that for every $p \geq 1$

$$
\begin{aligned}
\left(\int_{Q}\left\langle\nu_{y}^{x_{0}}, f^{p}\left(x_{0}, \cdot\right)\right\rangle \mathrm{d} y\right)^{1 / p} & \leq\left(\left\langle\mu_{x_{0}}, f^{p}\left(x_{0}, \cdot\right)\right\rangle+\mathcal{L}^{N}\left(Q \backslash Q^{\prime}\right)\left\|f^{p}\left(x_{0}, \cdot\right)\right\|_{L^{\infty}(B(0,3 K))}\right)^{1 / p} \\
& \leq \operatorname{esssup}_{x \in \Omega}\left(\mu_{x^{-}} \operatorname{ess~sup}_{\Sigma \in \mathbb{M}^{d \times N}} f(x, \Sigma)\right)+\mathcal{L}^{N}\left(Q \backslash Q^{\prime}\right)^{1 / p}\left\|f\left(x_{0}, \cdot\right)\right\|_{L^{\infty}(B(0,3 K))}
\end{aligned}
$$

where $K=\sup _{p}\left\|V_{p}\right\|_{\infty}$.

Let $\Omega^{\prime}=\{x \in \Omega \backslash N: f(x, \cdot)$ is $\mathcal{A}$-Young quasiconvex $\}$. Note that $\mathcal{L}^{N}\left(\Omega \backslash \Omega^{\prime}\right)=0$. We now consider $x_{0} \in \Omega^{\prime}$ and $\left(\bar{W}_{n}\right)$ constructed as above. Then, letting in (4.32) first $Q^{\prime} \rightarrow Q$ and then $p \rightarrow+\infty$, by ([3], Lem. 4.5), we obtain

$$
\underset{y \in Q}{\operatorname{ess} \sup }\left(\nu_{y}^{x_{0}}-\underset{\Sigma \in \mathbb{M}^{d \times N}}{\operatorname{ess} \sup _{0}} f\left(x_{0}, \Sigma\right)\right) \leq \underset{x \in \Omega}{\operatorname{ess} \sup }\left(\mu_{x^{-}} \underset{\Sigma \in \mathbb{M}^{d \times N}}{\operatorname{ess} \sup ^{d \times N}} f(x, \Sigma)\right) .
$$

By properties (1) and (3) we have that

$$
V\left(x_{0}\right)=\int_{\mathbb{M}^{d \times N}} \Sigma \mathrm{d} \nu_{y}^{x_{0}}(\Sigma)
$$

for a.e. $y \in \Omega$ (see [15], Rem. 2.3(ii)). Moreover, since $\mu=\left(\mu_{y}\right)_{y \in Q}$ is $\mathcal{A}$ - $\infty$ Young measure and $f\left(x_{0}, \cdot\right)$ is $\mathcal{A}$-Young quasiconvex we have that

$$
f\left(x_{0}, V\left(x_{0}\right)\right)=f\left(x_{0}, \int_{\mathbb{M}^{d \times N}} \Sigma \mathrm{d} \nu_{y}^{x_{0}}(\Sigma)\right) \leq \underset{y \in Q}{\operatorname{ess} \sup }\left(\nu_{y}^{x_{0}}-\underset{\Sigma \in \mathbb{M}^{d \times N}}{\operatorname{ess} \sup } f\left(x_{0}, \Sigma\right)\right)
$$

for every $x_{0} \in \Omega^{\prime}$. By (4.33) we get then (4.31) which concludes the proof of the liminf inequality. Since $F_{p} \leq|\Omega|^{1 / p} F$ for every $p \geq 1$, by the liminf inequality we get also the sequential lower semincontinuity of the functional $F$.

Theorem 4.2 (Sufficient condition). Let $f: \Omega \times \mathbb{M}^{d \times N} \rightarrow[-\infty,+\infty]$ be a Borel function, $f \not \equiv+\infty$. Assume that $f(x, \cdot)$ is lower semicontinuous and $\mathcal{A}$-Young quasiconvex for a.e. $x \in \Omega$. Then the functional $F: L^{\infty}\left(\Omega ; \mathbb{M}^{d \times N}\right) \cap \operatorname{Ker} \mathcal{A} \rightarrow \mathbb{R}$ defined by (4.27) is sequentially lower semicontinuous with respect to the $L^{\infty}$-weak* convergence.

Proof. The proof is achieved in two steps.

Step 1. We first deal with $f$ nonnegative and such that $f(x, \cdot)$ is locally bounded for a.e. $x \in \Omega$. For every fixed $n \in \mathbb{N}$ we define

$$
f_{n}(x, \Sigma):=f(x, \Sigma) \vee \frac{1}{n}|\Sigma|
$$


for every $(x, \Sigma) \in \Omega \times \mathbb{M}^{d \times N}$. We can easily check that the function $f_{n}$ is $\mathcal{A}$-Young quasiconvex, lower semicontinuous, locally bounded in the second variable. Moreover, it satisfies the growth condition (4.28) with $\alpha=1 / n$ for every fixed $n \in \mathbb{N}$. We now define the functional $F(n): L^{\infty}\left(\Omega ; \mathbb{M}^{d \times N}\right) \cap \operatorname{Ker} \mathcal{A} \rightarrow \mathbb{R}$ given by

$$
F(n)(V):=\operatorname{ess}_{x \in \Omega} f_{n}(x, V(x)) .
$$

By Theorem 4.1, $F(n)$ is sequentially $L^{\infty}$-weakly* lower semicontinuous.

Let $\left(V_{k}\right) \in L^{\infty}\left(\Omega ; \mathbb{M}^{d \times N}\right) \cap \operatorname{Ker} \mathcal{A}$ be a sequence $L^{\infty}$-weakly* converging to $V$. Without loss of generality we may assume that $\liminf _{k \rightarrow \infty} F\left(V_{k}\right)=\lim _{k \rightarrow \infty} F\left(V_{k}\right)$. Let $M:=\sup _{k}\left\|V_{k}\right\|_{\infty}$. If $\lim _{k \rightarrow \infty} F\left(V_{k}\right)>0$ then there exists $n_{0}, k_{0} \in \mathbb{N}$ such that

$$
F\left(V_{k}\right) \geq \frac{M}{n_{0}}
$$

for every $k \geq k_{0}$. It implies that

$$
F\left(V_{k}\right) \geq \frac{1}{n}\left\|V_{k}\right\|_{\infty}
$$

for every $n \geq n_{0}$ and $k \geq k_{0}$. We remark that

$$
F(n)(V)=F(V) \vee \frac{1}{n}\|V\|_{\infty},
$$

and that the sequence $(F(n))$ converges pointwise to the functional $F$ as $n \rightarrow \infty$ (see [18], Prop. 4.3).

In particular, we find that

$$
F(n)\left(V_{k}\right)=F\left(V_{k}\right) \vee \frac{1}{n}\left\|V_{k}\right\|_{\infty}=F\left(V_{k}\right)
$$

for every $k \geq k_{0}$ and $n \geq n_{0}$. By the lower semicontinuity of $F(n)$ we have that

$$
F(n)(V) \leq \liminf _{k \rightarrow \infty} F(n)\left(V_{k}\right)=\lim _{k \rightarrow \infty} F\left(V_{k}\right)
$$

for every $n \geq n_{0}$. Passing to the limit as $n \rightarrow \infty$ we obtain

$$
F(V) \leq \liminf _{k \rightarrow \infty} F\left(V_{k}\right)
$$

If $\lim _{k \rightarrow \infty} F\left(V_{k}\right)=0$, then we have that for every $n \in \mathbb{N}$

$$
F(V) \leq\left(F(V) \vee \frac{1}{n}\|V\|_{\infty}\right)=F(n)(V) \leq \liminf _{k \rightarrow \infty} F(n)\left(V_{k}\right)=\liminf _{k \rightarrow \infty}\left(F\left(V_{k}\right) \vee \frac{1}{n}\left\|V_{k}\right\|_{\infty}\right) \leq \frac{M}{n} .
$$

Passing to the limit as $n \rightarrow \infty$, we find that $F(V)=0$.

Step 2. We define $g(x, \Sigma):=\arctan (f(x, \Sigma))+\frac{\pi}{2}$. Since $f$ is an $\mathcal{A}$-Young quasiconvex function, by the monotonicity of the arctangent function, it is easy to see that $g$ is a nonnegative $\mathcal{A}$-Young quasiconvex function. Moreover, $g$ is bounded and lower semicontinuous with respect to the second variable. By Step 1, we have that the functional $G: L^{\infty}\left(\Omega ; \mathbb{M}^{d \times N}\right) \cap \operatorname{Ker} \mathcal{A} \rightarrow \mathbb{R}$ defined by

$$
G(V):=\operatorname{ess}_{x \in \Omega} \sup g(x, V(x))
$$

is sequentially $L^{\infty}$-weakly* lower semicontinuous. Let $\left(V_{k}\right) \in L^{\infty}\left(\Omega ; \mathbb{M}^{d \times N}\right) \cap \operatorname{Ker} \mathcal{A}$ be a sequence weakly* converging to $V$ and let $\left(V_{n_{k}}\right)$ be a subsequence such that

$$
\liminf _{k \rightarrow \infty} \operatorname{ess~sup}_{x \in \Omega} f\left(x, V_{k}(x)\right)=\lim _{n \rightarrow \infty} \operatorname{ess~sup}_{x \in \Omega} f\left(x, V_{k_{n}}(x)\right) .
$$


Since

$$
\liminf _{k \rightarrow \infty} \arctan \left(\underset{x \in \Omega}{\operatorname{essup} \sup }\left(f\left(x, V_{k}(x)\right)\right)\right) \geq \arctan \left(\liminf _{k \rightarrow \infty} \underset{x \in \Omega}{\operatorname{essips}} f\left(x, V_{k}(x)\right)\right)
$$

it easily follows that

$$
\liminf _{k \rightarrow \infty} \arctan \left(\underset{x \in \Omega}{\operatorname{ess} \sup }\left(f\left(x, V_{k}(x)\right)\right)\right)=\lim _{n \rightarrow \infty} \arctan \left(\underset{x \in \Omega}{\operatorname{ess} \sup } f\left(x, V_{k_{n}}(x)\right)\right) .
$$

By the lower semicontinuity of $\mathrm{G}$ we have that

$$
G(V) \leq \lim _{n \rightarrow \infty} G\left(V_{k_{n}}\right)
$$

and, consequently,

$$
\arctan \left(\underset{x \in \Omega}{\operatorname{ess} \sup _{0}} f(x, V(x))\right) \leq \lim _{n \rightarrow \infty} \arctan \left(\underset{x \in \Omega}{\operatorname{ess} \sup } f\left(x, V_{k_{n}}(x)\right)\right) .
$$

Gathering the previous inequalities, we can now conclude that

$$
\left.F(V)=\operatorname{ess}_{x \in \Omega} f(x, V(x))\right) \leq \lim _{n \rightarrow \infty} \operatorname{ess~sup}_{x \in \Omega} f\left(x, V_{k_{n}}(x)\right) \leq \lim _{k \rightarrow \infty} \operatorname{ess} \sup _{x \in \Omega} f\left(x, V_{k}(x)\right)=\liminf _{k \rightarrow \infty} F\left(V_{k}\right) .
$$

Remark 4.3. Let $f: \mathbb{M}^{d \times N} \mapsto[0,+\infty)$ be a lower semicontinuous function satisfying the growth condition

$$
f(\Sigma) \geq \alpha|\Sigma|
$$

for every $\Sigma \in \mathbb{M}^{d \times N}$ and a fixed $\alpha>0$. By (4.31) we have that

$$
f\left(\int_{\mathbb{M}^{d \times N}} \Sigma \mathrm{d} \mu_{x}(\Sigma)\right) \leq \underset{x \in \Omega}{\operatorname{ess} \sup _{x}} \mu_{x^{-}} \underset{\Sigma \in \mathbb{M}^{d \times N}}{\operatorname{ess} \sup } f(\Sigma)
$$

for a.e. $x \in \Omega$, for every $\mathcal{A}$ - $\infty$-Young measure $\mu=\left(\mu_{x}\right)_{x \in \Omega}$ and for every $\Omega$ open bounded subset of $\mathbb{R}^{N}$. In particular, $f$ is an $\mathcal{A}$-Young quasiconvex function and the condition (3.25) holds true not only for the unit cube $Q$ but for every open bounded subset of $\mathbb{R}^{N}$.

\section{SOME NECESSARY CONDITIONS FOR THE $w^{*}$-LOWER SEMICONTINUITY}

In the following theorem we provide necessary conditions for the lower semicontinuity of functionals as in (4.27). In particular, we prove that the $\mathcal{A}$-weak quasiconvexity is a necessary condition too. However, this condition is not sufficient as proved by a counter-example in [20]. The proof of (i) and (ii) closely follows that of ([7], Lem. 2.8) with the necessary changes.

Theorem 5.1 (Necessary conditions). Let $f: \Omega \times \mathbb{M}^{d \times N} \rightarrow \mathbb{R}$ be a Borel function. Assume that there exists a function $\omega:[0,+\infty) \times[0,+\infty) \mapsto[0,+\infty])$ continuous in its first variable, non-decreasing in its second variable, $\omega(0, t)=0$ for every $t>0$, and such that

$$
\left|f\left(x_{1}, \Sigma\right)-f\left(x_{2}, \Sigma\right)\right| \leq \omega\left(\left|x_{1}-x_{2}\right|,|\Sigma|\right)
$$

for every $x_{1}, x_{2} \in \mathbb{R}^{N}, \Sigma \in \mathbb{M}^{d \times N}$. Let $F: L^{\infty}\left(\Omega ; \mathbb{M}^{d \times N}\right) \cap \operatorname{Ker} \mathcal{A} \times \mathcal{O}(\Omega) \rightarrow \mathbb{R}$ be defined by

$$
F(V, B):=\operatorname{ess}_{x \in B} f(x, V(x)),
$$


for every $V \in L^{\infty}\left(\Omega ; \mathbb{M}^{d \times N}\right) \cap \operatorname{Ker} \mathcal{A}$ and $B \in \mathcal{O}(\Omega)$. Assume that $F(\cdot, B)$ is $L^{\infty}$-weakly lower semicontinuous for every $B \in \mathcal{O}(\Omega)$. Then

(i) $\forall x \in \mathbb{R}^{N}$

$$
\Sigma \mapsto f(x, \Sigma)
$$

is a lower semicontinuous function in $\mathbb{R}^{N}$;

(ii) $\forall x_{0} \in \Omega$ and for every $N$-cube $Y \subset \mathbb{R}^{N}$

$$
f\left(x_{0}, f_{Y} V(x) \mathrm{d} x\right) \leq \operatorname{ess~sup}_{x \in Y} f\left(x_{0}, V(x)\right)
$$

for every $V \in L_{\#}^{\infty}\left(Y ; \mathbb{M}^{d \times N}\right) \cap \operatorname{Ker} \mathcal{A}$. In particular, $f\left(x_{0}, \cdot\right)$ is $\mathcal{A}$-weak quasiconvex;

(iii) $\forall x \in \Omega$ and for every $t \in[0,1]$ it holds

$$
f\left(x, t \Sigma_{1}+(1-t) \Sigma_{2}\right) \leq \max \left\{f\left(x, \Sigma_{1}\right), f\left(x, \Sigma_{2}\right)\right\}
$$

for every $\left(\Sigma^{1}-\Sigma^{2}\right) \in \Lambda$, where

$$
\Lambda:=\bigcup_{w \in S^{N-1}} \operatorname{Ker} \mathbb{A}(w)
$$

Proof.

(i) Let us fix $x_{0} \in \mathbb{R}^{N}$ and let us consider $\Sigma_{h} \rightarrow \Sigma$ in $\mathbb{M}^{d \times N}$. We want to prove that $f\left(x_{0}, \Sigma\right) \leq$ $\liminf _{h \rightarrow \infty} f\left(x_{0}, \Sigma_{h}\right)$. Since $F$ is weak* lower semicontinuous we trivially have that

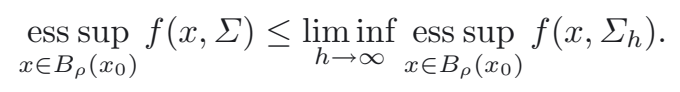

Since $\left(\Sigma_{h}\right)$ is bounded, by $(5.37)$, it follows that

$$
\begin{aligned}
f\left(x, \Sigma_{h}\right) & =f\left(x, \Sigma_{h}\right)-f\left(x_{0}, \Sigma_{h}\right)+f\left(x_{0}, \Sigma_{h}\right) \\
& \leq \omega\left(\left|x-x_{0}\right|,\left|\Sigma_{h}\right|\right)+f\left(x_{0}, \Sigma_{h}\right) \\
& \leq \omega\left(\left|x-x_{0}\right|, c\right)+f\left(x_{0}, \Sigma_{h}\right) .
\end{aligned}
$$

Then

$$
\operatorname{ess~sup}_{x \in B_{\rho}\left(x_{0}\right)} f\left(x, \Sigma_{h}\right) \leq f\left(x_{0}, \Sigma_{h}\right)+\operatorname{esssup}_{x \in B_{\rho}\left(x_{0}\right)} \omega\left(\left|x-x_{0}\right|, c\right)
$$

and, passing to the limit as $h \rightarrow \infty$, we find that

$$
\liminf _{h \rightarrow \infty} \operatorname{esssup}_{x \in B_{\rho}\left(x_{0}\right)} f\left(x, \Sigma_{h}\right) \leq \liminf _{h \rightarrow \infty} f\left(x_{0}, \Sigma_{h}\right)+\operatorname{ess}_{x \in B_{\rho}\left(x_{0}\right)} \omega\left(\left|x-x_{0}\right|, c\right) .
$$

It implies that

$$
\underset{x \in B_{\rho}\left(x_{0}\right)}{\operatorname{ess} \sup _{0}} f(x, \Sigma) \leq \liminf _{h \rightarrow \infty} f\left(x_{0}, \Sigma_{h}\right)+\operatorname{ess}_{x \in B_{\rho}\left(x_{0}\right)} \omega\left(\left|x-x_{0}\right|, c\right) .
$$

Passing to the limit as $\rho \rightarrow 0^{+}$we have that

$$
\begin{aligned}
f\left(x_{0}, \Sigma\right) & =\lim _{\rho \rightarrow 0^{+}} \operatorname{esssup}_{x \in B_{\rho}\left(x_{0}\right)} f(x, \Sigma) \\
& \leq \liminf _{h \rightarrow \infty} f\left(x_{0}, \Sigma_{h}\right)+\lim _{\rho \rightarrow 0^{+}} \operatorname{ess~sup}_{x \in B_{\rho}\left(x_{0}\right)} \omega\left(\left|x-x_{0}\right|, c\right),
\end{aligned}
$$

which concludes the proof of the lower semicontinuity of the function $f\left(x_{0}, \cdot\right)$. 
(ii) Let $V \in L_{\#}^{\infty}\left(Y ; \mathbb{M}^{d \times N}\right) \cap \operatorname{Ker} \mathcal{A}$. We define $V_{n}(x)=V\left(n\left(x-x_{0}\right)\right)$. Then, $V_{n} \in L^{\infty}\left(\Omega ; \mathbb{M}^{d \times N}\right) \cap \operatorname{Ker} \mathcal{A}$ is a $(1 / n) Y$-periodic function and it converges weakly* to $f_{Y} V(x) \mathrm{d} x$ in $L^{\infty}\left(\Omega ; \mathbb{M}^{d \times N}\right)$. By the lower semicontinuity of the functional $F$ we have that

$$
\underset{x \in B_{\rho}\left(x_{0}\right)}{\operatorname{ess} \operatorname{up}_{0}} f\left(x, f_{Y} V(y) \mathrm{d} y\right) \leq \liminf _{n \rightarrow \infty} \operatorname{ess~sup}_{x \in B_{\rho}\left(x_{0}\right)} f\left(x, V_{n}(x)\right) .
$$

Since $f$ is continuous in its first variable, we get the left hand side of (5.38) passing to the limit in (5.40) as $\rho \rightarrow 0^{+}$; i.e.,

$$
\lim _{\rho \rightarrow 0^{+}} \operatorname{ess}_{x \in B_{\rho}\left(x_{0}\right)} f\left(x, f_{Y} V(y) \mathrm{d} y\right)=f\left(x_{0}, f_{Y} V(y) \mathrm{d} y\right) .
$$

By the periodicity of $V_{n}$ and $n$ big enough we have that

$$
\underset{x \in B_{\rho}\left(x_{0}\right)}{\operatorname{ess} \sup _{0}} f\left(x_{0}, V_{n}(x)\right)=\underset{x \in Y}{\operatorname{ess} \sup _{0}} f\left(x_{0}, V(x)\right) .
$$

Hence, we can get the right hand side of inequality (5.38) computing the following limit

$$
\lim _{n \rightarrow \infty} \operatorname{esssup}_{x \in B_{\rho}\left(x_{0}\right)} f\left(x_{0}, V_{n}(x)\right)=\underset{x \in Y}{\operatorname{ess} \sup } f\left(x_{0}, V(x)\right) .
$$

On the other hand, by (5.37) we have that

$$
\left|f\left(x, V_{n}(x)\right)-f\left(x_{0}, V_{n}(x)\right)\right| \leq \omega\left(\left|x-x_{0}\right|,\left|V_{n}(x)\right|\right) \leq \omega\left(\left|x-x_{0}\right|, c\right) ;
$$

hence,

$$
\begin{aligned}
f\left(x, V_{n}(x)\right) & =f\left(x, V_{n}(x)\right)-f\left(x_{0}, V_{n}(x)\right)+f\left(x_{0}, V_{n}(x)\right) \\
& \leq \omega\left(\left|x-x_{0}\right|, c\right)+\underset{x \in B_{\rho}\left(x_{0}\right)}{\operatorname{ess} \sup _{0}} f\left(x_{0}, V_{n}(x)\right) .
\end{aligned}
$$

It implies that

$$
\underset{x \in B_{\rho}\left(x_{0}\right)}{\operatorname{ess} \sup _{0}} f\left(x, V_{n}(x)\right) \leq \operatorname{ess}_{x \in B_{\rho}\left(x_{0}\right)} \omega\left(\left|x-x_{0}\right|, c\right)+\underset{x \in B_{\rho}\left(x_{0}\right)}{\operatorname{ess} \sup } f\left(x_{0}, V_{n}(x)\right) .
$$

By (5.40) and gathering the previous inequalities we get that

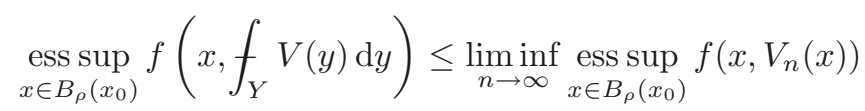

$$
\begin{aligned}
& \leq \liminf _{n \rightarrow \infty} \operatorname{esssup}_{x \in B_{\rho}\left(x_{0}\right)} f\left(x_{0}, V_{n}(x)\right)+\operatorname{ess}_{x \in B_{\rho}\left(x_{0}\right)} \omega\left(\left|x-x_{0}\right|, c\right) \\
& =\underset{x \in Y}{\operatorname{ess} \sup } f\left(x_{0}, V(x)\right)+\operatorname{ess}_{x \in B_{\rho}\left(x_{0}\right)} \omega\left(\left|x-x_{0}\right|, c\right) .
\end{aligned}
$$

Passing to the limit as $\rho \rightarrow 0^{+}$we get the formula (5.38).

Finally, for any $W \in L_{\#}^{\infty}\left(Y ; \mathbb{M}^{d \times N}\right) \cap \operatorname{Ker} \mathcal{A}$ with $\int_{Y} W(x) \mathrm{d} x=0$ we can define a function $V=\Sigma+W \in$ $L_{\#}^{\infty}\left(Y ; \mathbb{M}^{d \times N}\right) \cap \operatorname{Ker} \mathcal{A}$ satisfying $f_{Y} V(x) \mathrm{d} x=\Sigma$. Hence,

$$
f\left(x_{0}, \Sigma\right)=f\left(x_{0}, f_{Y} V(x) \mathrm{d} x\right) \leq \operatorname{essup}_{x \in Y} f\left(x_{0}, V(x)\right)=\underset{x \in Y}{\operatorname{ess} \sup } f\left(x_{0}, \Sigma+W(x)\right)
$$


for every fixed $x_{0} \in \Omega, \Sigma \in \mathbb{M}^{d \times N}$ and $W \in L_{\#}^{\infty}\left(Y ; \mathbb{M}^{d \times N}\right) \cap \operatorname{Ker} \mathcal{A}$ with $\int_{Y} W(x) \mathrm{d} x=0$. In particular, $f\left(x_{0}, \cdot\right)$ is $\mathcal{A}$-weak quasiconvex.

(iii) Let $\Sigma^{1}, \Sigma^{2} \in \mathbb{M}^{d \times N}$ and let $w \in S^{N-1}$ be a vector such that $\left(\Sigma^{1}-\Sigma^{2}\right) \in \operatorname{Ker} \mathbb{A}(w)$. We define

$$
V(x)= \begin{cases}(1-t)\left(\Sigma^{1}-\Sigma^{2}\right), & x \in A_{1} \\ -t\left(\Sigma^{1}-\Sigma^{2}\right), & x \in A_{2}\end{cases}
$$

where

$$
\begin{aligned}
& A_{1}=\left\{x \in \mathbb{R}^{N}: j<\langle x, w\rangle<j+t, j \in \mathbb{Z}\right\}, \\
& A_{2}=\left\{x \in \mathbb{R}^{N}: j+t<\langle x, w\rangle<j+1, j \in \mathbb{Z}\right\}
\end{aligned}
$$

for a fixed $t \in(0,1)$. Since $\left(\Sigma^{1}-\Sigma^{2}\right) \in \operatorname{Ker} \mathbb{A}(w)$, we may easily check that $\mathcal{A} V=0$ (see e.g. [2], Thm. 4.2, Step 3). Moreover, by construction $V \in L_{\#}^{\infty}\left(Y ; \mathbb{M}^{d \times N}\right)$ for a suitable cube $Y \subset \mathbb{R}^{N}$ and satisfies $\int_{Y} V \mathrm{~d} x=0$. Hence, by (5.41), for every fixed $x_{0} \in \Omega$ and for every $t \in[0,1]$ we have that

$$
\begin{aligned}
f\left(x_{0}, \Sigma\right) & \leq \underset{x \in Y}{\operatorname{essup} \sup } f\left(x_{0}, \Sigma+V(x)\right) \\
& \leq \underset{x \in A_{1} \cup A_{2}}{\operatorname{ess} \sup _{0}} f\left(x_{0}, \Sigma+V(x)\right) \\
& \left.=\underset{x \in A_{1}}{\max \{\operatorname{esssup}} f\left(x_{0}, \Sigma+(1-t)\left(\Sigma^{1}-\Sigma^{2}\right)\right), \underset{x \in A_{2}}{\operatorname{ess} \sup } f\left(x_{0}, \Sigma-t\left(\Sigma^{1}-\Sigma^{2}\right)\right)\right\} .
\end{aligned}
$$

In particular, for $\Sigma=t \Sigma^{1}+(1-t) \Sigma^{2}$ we get that

$$
f\left(x_{0}, t \Sigma^{1}+(1-t) \Sigma^{2}\right) \leq \max \left\{f\left(x_{0}, \Sigma^{1}\right), f\left(x_{0}, \Sigma^{2}\right)\right\} .
$$

Remark 5.2. Note that if $f: \mathbb{M}^{d \times N} \rightarrow \mathbb{R}$ is a lower semicontinuous and $\mathcal{A}$-Young quasiconvex function then, by Theorems 4.2 and 5.1, we have that $f$ satisfies conditions (5.38) and (5.39). In particular, $f$ is an $\mathcal{A}$-weak quasiconvex function and the condition (5.38) holds true for every $N$-cube $Y$.

\section{Div-Young AND curl-Young QUASICONVEXITY}

In this section we study the properties of the $\mathcal{A}$-Young quasiconvex functions in the divergence-free and curl-free case; that is, $\mathcal{A}=$ Div , curl .

As already remarked, in the gradient case, Barron, Jensen and Wang proved that strong Morrey quasiconvexity is a necessary and sufficient condition for the lower semicontinuity of supremal functionals with $f=f(\Sigma)$ lower semicontinuous (see [7], Def. 2.1, Thms. 2.6 and 2.7). Therefore, by Theorem 4.2 we have that a curl-Young quasiconvex function is, in particular, a strong Morrey quasiconvex function (see Prop. 6.1 below). Note that if $f$ depends explicitly on $x$, then strong Morrey quasiconvexity provides a necessary and sufficient condition under continuity assumption on $f(\cdot, \Sigma)$. Therefore, in the case of $\mathcal{A}=\operatorname{curl}$, Theorem 4.2 improves ([7], Thm. 2.6) since we do not require any continuity assumption on $f(\cdot, \Sigma)$.

Proposition 6.1. Let $f: \Omega \times \mathbb{M}^{d \times N} \rightarrow \mathbb{R}$ be a Borel function. Assume that $f(x, \cdot)$ is lower semicontinuous and curl-Young quasiconvex for a.e. $x \in \Omega$. Then, $f(x, \cdot)$ is a strong Morrey quasiconvex function for a.e. $x \in \Omega$.

Proof. Let $x \in \Omega$ be such that the function $f(x, \cdot)$ is lower semicontinuous and curl-Young quasiconvex. For every open set $A \subset \mathbb{R}^{N}$ let $F_{x}(\cdot, A): W^{1, \infty}\left(A ; \mathbb{M}^{d \times N}\right) \rightarrow \mathbb{R}$ be the functional defined by

$$
F_{x}(u, A):=\operatorname{esssup}_{y \in A} f(x, D u(y)) .
$$

Then, by Theorem 4.2 , we have that for every open set $A \subset \mathbb{R}^{N}$ the functional $F_{x}(\cdot, A)$ is sequentially $L^{\infty}$ weakly* lower semicontinuous on $W^{1, \infty}\left(A ; \mathbb{M}^{d \times N}\right)$. Thanks to the necessary condition in ([7], Thm. 2.7), it follows that $f(x, \cdot)$ is a strong Morrey quasiconvex function. 
In Proposition 6.3 we prove that polylevelconvex functions (see Def. 6.2 below) are curl-Young quasiconvex functions. In ([7], Cor. 3.9) they show that a lower semicontinuous polylevelconvex function is a strong Morrey quasiconvex function.

Definition 6.2 ([7], Def. 3.5). A measurable function $f: \mathbb{M}^{d \times N} \rightarrow \mathbb{R}$ is called polylevelconvex if there exists a level convex function $g: \mathbb{R}^{c(N, d)} \rightarrow \mathbb{R}$ such that $\left.f(\Sigma)=(g \circ T)(\Sigma)\right)$ where $c(N, d)$ is given by

$$
c(N, d)=\sum_{s=1}^{\min (N, d)} \frac{d ! N !}{(s !)^{2}(N-s) !(d-s) !}
$$

and $T: \mathbb{M}^{d \times N} \rightarrow \mathbb{R}^{c(N, d)}$ is the map consisting of $\Sigma$ and all of its $s \times s$ minors for $s \leq \min (N, d)$.

Note that such functions have been referred in [7] as polyquasiconvex functions.

Proposition 6.3. Let $g: \mathbb{R}^{c(N, d)} \rightarrow[0,+\infty)$ be a lower semicontinuous and level convex function. Then the polylevelconvex function $f=g \circ T$ is curl-Young quasiconvex.

Proof. The proof is achieved in two steps.

Step 1. We first deal with $g$ continuous function. We define $g_{n}(\xi):=g(\xi) \vee \frac{1}{n}|\xi|$. Then $g_{n}$ is a continuous and level convex function satisfying the growth condition (4.35). By ([3], Prop. 5.7) the polylevelconvex function $f_{n}(\Sigma):=g_{n} \circ T(\Sigma)=g(T(\Sigma)) \vee \frac{1}{n}|T(\Sigma)|$ is curl- $\infty$ quasiconvex. By Proposition $3.4 f_{n}$ is also a curl-Young quasiconvex function. In particular, for every curl- $\infty$-Young measure $\mu=\left(\mu_{x}\right)_{x \in Q}$ and for every $n \in \mathbb{N}$ we have that

$$
f\left(\int_{\mathbb{M}^{d \times N}} \Sigma \mathrm{d} \mu_{x}(\Sigma)\right) \leq f_{n}\left(\int_{\mathbb{M}^{d \times N}} \Sigma \mathrm{d} \mu_{x}(\Sigma)\right) \leq \underset{x \in Q}{\operatorname{ess} \sup } \mu_{x^{-}} \underset{\Sigma \in \mathbb{M}^{d \times N}}{\operatorname{ess} \sup _{n}} f_{n}(\Sigma) .
$$

Note that, since the sequence of functions generating the Young measure $\mu=\left(\mu_{x}\right)_{x \in Q}$ is bounded in $L^{\infty}\left(Q, \mathbb{M}^{d \times N}\right)$, by the Fundamental Theorem on Young Measure (see e.g. ([15], Thm. $\left.2.2(2)\right)$ ), there exists a compact set $K \subset \mathbb{M}^{d \times N}$ such that

$$
\operatorname{supp} \mu_{x} \subset K \quad \text { for a.e. } x \in Q \text {. }
$$

Therefore, we have that

$$
\underset{x \in Q}{\operatorname{ess} \sup } \mu_{x^{-}} \underset{\Sigma \in \mathbb{M}^{d \times N}}{\operatorname{ess} \sup ^{d \times N}}|T(\Sigma)|=\underset{x \in Q}{\operatorname{ess} \sup } \mu_{x^{-}} \underset{\Sigma \in K}{\operatorname{ess} \sup }|T(\Sigma)| \leq \max _{\Sigma \in K}|T(\Sigma)|=C \in \mathbb{R} .
$$

In addition,

$$
\underset{x \in Q}{\operatorname{ess} \sup } \mu_{x^{-}} \underset{\Sigma \in \mathbb{M}^{d \times N}}{\operatorname{ess} \sup _{n}} f_{n}(\Sigma)=\underset{x \in Q}{\operatorname{ess} \sup } \mu_{x^{-}} \underset{\Sigma \in \mathbb{M}^{d \times N}}{\operatorname{ess} \sup } g(T(\Sigma)) \vee \frac{1}{n} \underset{x \in Q}{\operatorname{ess} \sup } \mu_{x^{-}} \underset{\Sigma \in \mathbb{M}^{d \times N}}{\operatorname{ess} \sup }|T(\Sigma)| .
$$

We denote by $M=\operatorname{ess} \sup _{x \in Q} \mu_{x^{-}} \operatorname{ess} \sup _{\Sigma \in \mathbb{M}^{d \times N}} f(\Sigma)$. By (6.44) and (6.45), we have that

$$
\underset{x \in Q}{\operatorname{ess} \sup } \mu_{x^{-}} \underset{\Sigma \in \mathbb{M}^{d \times N}}{\operatorname{ess} \sup _{n}} f_{n}(\Sigma) \leq M \vee \frac{C}{n}
$$

for every $n \in \mathbb{N}$. Therefore, passing to the limit as $n \rightarrow \infty$, we get that

$$
\underset{x \in Q}{\operatorname{ess} \sup } \mu_{x^{-}} \underset{\Sigma \in \mathbb{M}^{d \times N}}{\operatorname{ess} \sup ^{d \times N}} f_{n}(\Sigma) \leq M .
$$

By (6.43) we get the thesis. 
Step 2. We now assume that $g$ is a lower semicontinuous function. Reasoning as in the proof of Proposition 2.9 we have that $g=\sup _{\lambda>0} g_{\lambda}$ where $g_{\lambda}$ is a continuous and level convex function given by the formula

$$
g_{\lambda}(\xi):=\inf \left\{g(\eta) \vee \lambda|\xi-\eta|: \eta \in \mathbb{R}^{c(N, d)}\right\} .
$$

By Step 1, we have that $g_{\lambda} \circ T$ is a curl-Young quasiconvex function, for every $\lambda>0$. Since $f(\Sigma)=(g \circ T)(\Sigma)=$ $\sup \left\{\left(g_{\lambda} \circ T\right)(\Sigma): \lambda>0\right\}$, we can conclude that $f$ is a curl-Young quasiconvex function.

Proposition 6.4. Let $f: \mathbb{M}^{d \times N} \rightarrow \mathbb{R}$ be a lower semicontinuous function.

(1) $(\mathcal{A}=\operatorname{div}, d=1)$. Let $f$ be a $\operatorname{div}-$ Young quasiconvex function. Then $f$ is a level convex function; i.e., $f$ satisfies

$$
f\left(x, t \Sigma_{1}+(1-t) \Sigma_{2}\right) \leq \max \left\{f\left(x, \Sigma_{1}\right), f\left(x, \Sigma_{2}\right)\right\},
$$

for every $\Sigma_{1}, \Sigma_{2} \in \mathbb{M}^{d \times N}$ and $t \in[0,1]$.

(2) $(\mathcal{A}=\operatorname{Div}, d \geq N>1)$. Let $f$ be a Div-Young quasiconvex function. Then $f$ is rank- $(N-1)$ level convex; i.e., $f$ satisfies (6.46) for every $\Sigma_{1}, \Sigma_{2} \in \mathbb{M}^{d \times N}$ with $\operatorname{rank}\left(\Sigma^{1}-\Sigma^{2}\right) \leq(N-1)$ and $t \in[0,1]$.

(3) $\left(\mathcal{A}=\right.$ curl). Let $f: \mathbb{M}^{d \times N} \mapsto \mathbb{R}$ be curl-Young quasiconvex. Then $f$ is rank-1 level convex; i.e., $f$ satisfies (6.46) for every $\Sigma_{1}, \Sigma_{2} \in \mathbb{M}^{d \times N}$ with $\operatorname{rank}\left(\Sigma^{1}-\Sigma^{2}\right) \leq 1$ and $t \in[0,1]$. In particular, if either $d=1$ or $N=1$ then $f$ is level convex.

Proof.

(1) If $d=1$ and $\mathcal{A}=\operatorname{div}$, then

$$
\operatorname{Ker} \mathbb{A}(w)=\left\{\xi \in \mathbb{R}^{N}:\langle\xi, w\rangle=0\right\}
$$

for every $w \in S^{N-1}$, which implies that $\Lambda=\mathbb{R}^{N}$. Note that, since for every $\xi^{1} \neq \xi^{2}$ there always exists $w \in S^{N-1}$ such that $\left\langle\xi^{1}-\xi^{2}, w\right\rangle=0$, we have in particular that $\left(\xi^{1}-\xi^{2}\right) \in \Lambda$. Hence, by Theorem 5.1, $f$ satisfies (6.46) for every $\xi^{1}, \xi^{2} \in \mathbb{R}^{N}$; i.e., $f$ is level convex.

(2) We recall that if $d>1$, we define $\operatorname{Div} V: \Omega \mapsto \mathbb{R}^{d}$ such that $(\operatorname{Div} V)_{i}=\operatorname{div}(V)_{i}$ whenever $i=1, \ldots, d$. Therefore the vectorial case, $d \geq N>1$, can be studied by generalising the previous case $d=1$. More precisely, we can prove that if $\left(\Sigma^{1}-\Sigma^{2}\right) \in \Lambda$ then $\operatorname{rank}\left(\Sigma^{1}-\Sigma^{2}\right) \leq(N-1)$. By Theorem 5.1 we have that $f$ satisfies (6.46) for every $\Sigma^{1} \neq \Sigma^{2} \in \mathbb{M}^{d \times N}$ with $\operatorname{rank}\left(\Sigma^{1}-\Sigma^{2}\right) \leq(N-1)$.

(3) If $\mathcal{A}=$ curl, then

$$
\operatorname{Ker} \mathbb{A}(w)=\left\{\xi \otimes w \in \mathbb{M}^{d \times N}: \xi \in \mathbb{R}^{d}, w \in S^{N-1}\right\} .
$$

By Theorem 5.1 we have that $f$ satisfies (6.46) along any rank-one directions.

Proposition 6.5. Let $f: \mathbb{M}^{d \times N} \rightarrow \mathbb{R}$ be a lower semicontinuous function.

(1) If $d=1$ then $f$ is level convex $\Longleftrightarrow \operatorname{div-Young~quasiconvex.~}$

(2) If either $d=1$ or $N=1$ then $f$ is level convex $\Longleftrightarrow$ curl-Young quasiconvex $\Longleftrightarrow$ strong Morrey quasiconvex.

Proof. For any constant-rank operator $\mathcal{A}$, by Proposition 3.3(2), we find that if $f$ is a level convex function then $f$ is also a $\mathcal{A}$-Young quasiconvex function. In order to get the equivalence in the div and curl free cases it is sufficient to apply Proposition 6.4. Moreover, by ([7], Cor. 3.9) we have that level convexity is equivalent to strong Morrey quasiconvexity which concludes also the proof of (2). 


\subsection{Examples}

In Propositions 3.3 and 3.4 we prove that $\mathcal{A}$-quasiconvex functions, level convex functions and $\mathcal{A}$ - $\infty$ quasiconvex functions belong to the class of $\mathcal{A}$-Young quasiconvex functions. Here we exhibit some examples (in the case of $\mathcal{A}=$ curl) in order to prove that the aforesaid classes are strictly included. We recall that a function is curl-quasiconvex if and only if it is quasiconvex.

Example 6.6. The function

$$
f(\Sigma)=\arctan (\operatorname{det} \Sigma), \quad \Sigma \in \mathbb{M}^{2 \times 2}
$$

is polylevelconvex, curl-Young quasiconvex but it is not level convex. In fact,

(1) let $g: \mathbb{R}^{5} \rightarrow[0,+\infty)$ be defined as $g(\xi)=\arctan \xi_{5}$ and let $T(\Sigma)=\left(\Sigma_{11}, \Sigma_{12}, \Sigma_{21}, \Sigma_{22}\right.$, det $\left.\Sigma\right)$ for every $\Sigma \in \mathbb{M}^{2 \times 2}$. Since $g$ is a level convex function, by Definition 6.2 we have that $f=g \circ T$ is a polylevelconvex function. Moreover, by Proposition 6.3 we have that $f$ is also a curl-Young quasiconvex function;

(2) let $\Sigma_{1}:=\left(\begin{array}{ll}1 & 0 \\ 0 & 0\end{array}\right)$, and $\Sigma_{2}:=\left(\begin{array}{ll}0 & 0 \\ 0 & 1\end{array}\right)$. For every $\lambda \in(0,1)$ we have that $\arctan \operatorname{det}\left(\lambda \Sigma_{1}+(1-\lambda) \Sigma_{2}\right)=$ $\arctan (\lambda(1-\lambda))>0$ while $\arctan \left(\operatorname{det} \Sigma_{1}\right)=\arctan \left(\operatorname{det} \Sigma_{2}\right)=0$. More precisely, there exists $\lambda=1 / 2$ such that

$$
f\left(\frac{1}{2} \Sigma_{1}+\frac{1}{2} \Sigma_{2}\right)=\arctan \frac{1}{4}>0>f\left(\Sigma_{1}\right) \vee f\left(\Sigma_{2}\right) .
$$

Therefore we may conclude that $f$ is not level convex.

Example 6.7. The function $f: \mathbb{M}^{2 \times 2} \rightarrow[0,+\infty)$ defined as

$$
f(\Sigma):=k(\Sigma) \vee h(|\Sigma|)
$$

with $k(\Sigma):=\arctan (\operatorname{det} \Sigma)$ and

$$
h(t):=\left\{\begin{array}{cl}
0 & \text { if } t \leq 1 \\
t-1 & \text { if } 1 \leq t \leq 2 \\
1 & \text { if } t \geq 2
\end{array}\right.
$$

is a curl-Young quasiconvex function, but not level convex and neither curl- $\infty$ quasiconvex nor quasiconvex. In fact,

(1) $h$ is a level convex function and by Example 6.6 we have that $k$ is a curl-Young quasiconvex function. Therefore $f$ is a curl-Young quasiconvex function since it is the maximum between two curl-Young quasiconvex functions;

(2) let $\Sigma_{1}$ and $\Sigma_{2}$ be as in Example 6.6; hence,

$$
f\left(\frac{1}{2} \Sigma_{1}+\frac{1}{2} \Sigma_{2}\right)=\arctan \frac{1}{4} \vee h\left(\frac{1}{\sqrt{2}}\right)=\arctan \frac{1}{4}>0=f\left(\Sigma_{1}\right) \vee f\left(\Sigma_{2}\right) .
$$

It follows that $f$ is not a level convex function;

(3) note that $f_{p}^{p}=\mathcal{Q}_{\text {curl }} f^{p}$ which coincides with the quasiconvex envelope of $f^{p}$. Since $f^{p}$ is also bounded, we have that $f_{p}^{p}=0$ (see [10], Ex. 4.2). Passing to the limit as $p \rightarrow+\infty$ we get that $\mathcal{Q}_{\text {curl }}^{\infty} f=0$; i.e., $f(\Sigma)>\mathcal{Q}_{\text {curl }}^{\infty} f(\Sigma)$, for every $\Sigma \in \mathbb{M}^{d \times N}$ with $|\operatorname{det} \Sigma|>0$. Therefore $f$ is not a curl- $\infty$ quasiconvex function;

(4) reasoning by contradiction we assume that $f$ is a quasiconvex function. Hence,

$$
g(\lambda):=f\left(\lambda \Sigma_{1}\right)
$$

is convex. Since $g(\lambda)=k\left(\lambda \Sigma_{1}\right) \vee h\left(\lambda\left|\Sigma_{1}\right|\right)=h\left(\lambda\left|\Sigma_{1}\right|\right)=h\left(\frac{\lambda}{2}\right)$ we get the contradiction. Note that we may also deduce that $f$ is not quasiconvex by ([3], Prop. 3.4(1)). 


\subsection{An application to the effective strenght for resistive materials}

In this section we characterise, by way of $\Gamma$-convergence, the effective strength set $K_{\text {eff }}$ in the context of electrical resistivity. More precisely, we consider

$$
K_{\text {eff }}=\left\{\xi \in \mathbb{R}^{N}: \exists \sigma \in L^{\infty}\left(Q ; \mathbb{R}^{N}\right), \int_{Q} \sigma \mathrm{d} x=0, \operatorname{div} \sigma=0, f(x, \xi+\sigma(x)) \leq 1 \text { a.e. } x \in Q\right\}
$$

and we assume that $f(x, \cdot)$ is a div-Young quasiconvex and lower semicontinuous function. Note that, by ([3], Prop. 5.3), div-Young quasiconvexity is equivalent to level convexity. The following theorem is an application of Theorem 2.10 and generalises both ([8], Props. 6.1, 6.2) and ([3], Thm. 6.1) where continuity and div $-\infty$ quasiconvex assumptions are considered.

Theorem 6.8. Let $f: Q \times \mathbb{R}^{N} \mapsto \mathbb{R}$ be a Borel function such that $f(x, \cdot)$ is lower semicontinuous and level convex for a.e. $x \in \Omega$ and satisfies the growth condition (4.28). For any $\xi \in \mathbb{R}^{N}$ let

$$
j_{p}^{\mathrm{eff}}(\xi):=\inf \left\{\left(\int_{Q} f^{p}(x, \xi+\sigma(x)) \mathrm{d} x\right)^{1 / p}: \sigma \in L^{p}\left(Q ; \mathbb{R}^{N}\right), \int_{Q} \sigma \mathrm{d} x=0, \operatorname{div} \sigma=0\right\} .
$$

Then, for any $\xi \in \mathbb{R}^{N}, j_{p}^{\text {eff }}(\xi)$ converges to $j_{\infty}^{\text {eff }}(\xi)$ given by

$$
j_{\infty}^{\mathrm{eff}}(\xi):=\inf \left\{\underset{x \in Q}{\operatorname{ess} \sup } f(x, \xi+\sigma(x)): \sigma \in L^{\infty}\left(Q ; \mathbb{R}^{N}\right), \int_{Q} \sigma \mathrm{d} x=0, \operatorname{div} \sigma=0\right\} .
$$

Moreover, the set $K_{\mathrm{eff}}$ is described by

$$
K_{\mathrm{eff}}=\left\{\xi \in \mathbb{R}^{N}: j_{\infty}^{\mathrm{eff}}(\xi) \leq 1\right\} .
$$

Proof. By Theorem 2.10, for every fixed $\xi \in \mathbb{R}^{N}$, we have that the functional $H_{p}: L^{1}\left(Q ; \mathbb{R}^{N}\right) \rightarrow \mathbb{R} \cup\{+\infty\}$ given by

$$
H_{p}(\sigma):=\left\{\begin{array}{cl}
\left(\int_{Q} f^{p}(x, \sigma(x)+\xi) \mathrm{d} x\right)^{1 / p} & \text { if } \sigma \in L^{p}\left(Q ; \mathbb{R}^{N}\right), \operatorname{div} \sigma=0, \int_{Q} \sigma \mathrm{d} x=0 \\
+\infty & \text { otherwise }
\end{array}\right.
$$

$\Gamma\left(w-L^{1}\right)$ - converges to the functional

$$
H(\sigma)=\left\{\begin{array}{cl}
\underset{x \in Q}{\operatorname{ess} \sup } f(x, \sigma(x)+\xi) & \text { if } \sigma \in L^{\infty}\left(Q ; \mathbb{R}^{N}\right), \operatorname{div} \sigma=0, \int_{Q} \sigma \mathrm{d} x=0 \\
+\infty & \text { otherwise. }
\end{array}\right.
$$

By assumption (4.28), for a fixed $q>1$, for every $p \geq q$ and for every $\lambda \in \mathbb{R}$ we get the following inclusion

$$
E_{\lambda, p}:=\left\{u \in L^{1}\left(\Omega ; \mathbb{R}^{N}\right): H_{p}(\sigma) \leq \lambda\right\} \subset\left\{u \in L^{1}\left(\Omega ; \mathbb{R}^{N}\right): \int_{\Omega}|\sigma|^{q} \mathrm{~d} x \leq C(q, \lambda)\right\}=: K_{\lambda} .
$$

Since $K_{\lambda}$ is closed and sequentially compact with respect to the $L^{1}$-weak topology, it follows that the sequence $\left(H_{p}\right)_{p \geq q}$ is equicoercive with respect to the same topology (see [14], Def. 7.6). Therefore, by ([14], Thm. 7.8), we find that

$$
\lim _{p \rightarrow \infty} j_{p}^{\mathrm{eff}}(\xi)=j_{\infty}^{\mathrm{eff}}(\xi)
$$

for any $\xi \in \mathbb{R}^{N}$. Since $f$ is a level convex function, the functional $H$ is weakly* lower semicontinuous on $L^{\infty}\left(Q ; \mathbb{R}^{N}\right)$ and the proof of (6.48) follows as in ([3], Thm. 6.1). 
Acknowledgements. The first author gratefully acknowledges the hospitality of the Department of Mathematical Sciences at University of Bath (UK) where this work was completed while she was visiting professor. Her research has been supported by a Marie Curie Intra-European Fellowship (IEF, 7th Framework Programme for Research), Proposal number 326044, MIDAS. The research of the second author was partially supported by GNAMPA 2014 under the project "Problemi di regolarità e teoria geometrica della misura in spazi metrici".

\section{REFERENCES}

[1] E. Acerbi, G. Buttazzo and F. Prinari, The class of functionals which can be represented by a supremum. J. Convex Anal. 9 (2002) 225-236.

[2] N. Ansini and A. Garroni, $\Gamma$-convergence of Functionals on Divergence Free Fields. ESAIM: COCV 13 (2007) 809-828.

[3] N. Ansini and F. Prinari, Power-law approximation under differential constraint. SIAM J. Math. Anal. 46 (2014) $1085-1115$.

[4] J.M. Ball, A version of the fundamental theorem for Young measures. PDE's and Continuum Models of Phase Transitions. Edited by M. Rascle, D. Serre and M. Slemrod. In vol. 344 of Lect. Notes Phys. Springer-Verlag, Berlin (1989) $207-215$.

[5] E.N. Barron and W. Liu, Calculus of Variation in $L^{\infty}$. Appl. Math. Optim. 35 (1997) 237-263.

[6] E.N. Barron, R. Jensen and W. Liu, Hopf-Lax type formula for $u_{t}+H(u, d u)=0$. J. Differ. Eq. 126 (1996) $48-61$.

[7] E.N. Barron, R. Jensen and C.Y. Wang, Lower semicontinuity of $L^{\infty}$ functionals. Ann. Inst. Henri Poincaré 4 (2001) $495-517$.

[8] M. Bocea and V. Nesi, $\Gamma$-convergence of power-law functionals, variational principles in $L^{\infty}$ and applications. SIAM J. Math. Anal. 39 (2008) 1550-1576.

[9] H. Berliocchi and J.M. Lasry, Intégral normales et mesures paramétrées en calcul des variations. Bull. Soc. Math. France 101 (1973) 129-184.

[10] A. Braides and A. Defranceschi, Homogenization of Multiple Integrals. Oxford University Press, Oxford (1998).

[11] A. Braides, I. Fonseca and G. Leoni, A-Quasiconvexity: Relaxation and Homogenization. ESAIM: COCV 5 (2000) 539-577.

[12] P. Cardaliaguet and F. Prinari, Supremal representation of $L^{\infty}$ functionals. Appl. Math. Optim. 52 (2005) 129-141.

[13] T. Champion, L. De Pascale and F. Prinari, $\Gamma$-convergence and absolute minimizers for supremal functionals. ESAIM: COCV 10 (2004) 14-27.

[14] G. Dal Maso, An Introduction to $\Gamma$-convergence. Birkhäuser, Boston (1993).

[15] I. Fonseca and S. Müller, A-Quasiconvexity, lower semicontinuity and Young measures. SIAM J. Math. Anal. 30 (1999) 1355-1390.

[16] A. Garroni, V. Nesi and M. Ponsiglione, Dielectric breakdown: optimal bounds. Proc. Roy. Soc. London A 457 (2001) 23172335.

[17] F. Murat, Compacité par compensation: condition necessaire et suffisante de continuité faible sous une hypothése de rang constant. Ann. Scuola Norm. Sup. Pisa Cl. Sci. 8 (1981) 68-102.

[18] F. Prinari, Semicontinuity and supremal representation in the Calculus of Variations. Appl. Math. Optim. 54 (2008) 111-145.

[19] F. Prinari, Semicontinuity and relaxation of $L^{\infty}$-functionals. Adv. Calc. Var. 2 (2009), 43-71.

[20] F. Prinari, On the necessary condition for the lower semicontinuity of supremal functionals. In preparation.

[21] A. Ribeiro and E. Zappale, Existence of minimizers for non-level convex supremal functionals. SIAM J. Control Optim. 52 (2014) 3341-3370.

[22] L. Tartar, Compensated compactness and applications to partial differential equations. Nonlinear Analysis and Mechanics: Heriot-Watt Symposium. Edited by R. Knops. Longman, Harlow. Pitman Res. Notes Math. Ser. 39 (1979) $136-212$. 